\title{
On uniform exponential ergodicity of Markovian multiclass many-server queues in the Halfin-Whitt regime
}

\author{
Ari Arapostathis, Hassan Hmedi \\ Department of Electrical and Computer Engineering, The University of Texas at Austin, \\ 2501 Speedway, EER 7.824, Austin, TX 78712, [ari,hmedi]@utexas.edu \\ Guodong Pang \\ The Harold and Inge Marcus Dept. of Industrial and Manufacturing Eng., College of Engineering, \\ Pennsylvania State University, University Park, PA 16802, gup3@psu.edu
}

\begin{abstract}
We study ergodic properties of Markovian multiclass many-server queues which are uniform over scheduling policies, as well as the size of the system. The system is heavily loaded in the Halfin-Whitt regime, and the scheduling policies are work-conserving and preemptive. We provide a unified approach via a Lyapunov function method that establishes Foster-Lyapunov equations for both the limiting diffusion and the prelimit diffusion-scaled queueing processes simultaneously.

We first study the limiting controlled diffusion, and show that if the spare capacity (safety staffing) parameter is positive, the diffusion is exponentially ergodic uniformly over all stationary Markov controls, and the invariant probability measures have uniform exponential tails. This result is sharp, since when there is no abandonment and the spare capacity parameter is negative, then the controlled diffusion is transient under any Markov control. In addition, we show that if all the abandonment rates are positive, the invariant probability measures have sub-Gaussian tails, regardless whether the spare capacity parameter is positive or negative.

Using the above results, we proceed to establish the corresponding ergodic properties for the diffusionscaled queueing processes. In addition to providing a simpler proof of the results in Gamarnik and Stolyar [Queueing Syst (2012) 71:25-51], we extend these results to the multiclass models with renewal arrival processes, albeit under the assumption that the mean residual life functions are bounded. For the Markovian model with Poisson arrivals, we obtain stronger results and show that the convergence to the stationary distribution is at an exponential rate uniformly over all work-conserving stationary Markov scheduling policies.

Key words: multiclass many-server queues, Halfin-Whitt (QED) regime, exponential ergodicity, diffusion scaling

MSC2000 subject classification: 90B22, 60K25, 90B15
\end{abstract}

1. Introduction. Multiclass many-server queues in the Halfin-Whitt $(\mathrm{H}-\mathrm{W})$ regime have been extensively studied as a useful model for large-scale service systems. In this paper we focus on ergodic properties of such multiclass queueing models. The ergodic properties of these systems have been the subject of great interest in applied probability (for a discussion see [20,33, 34, 35, $32,16])$. It is important to understand if a queueing system is stable and the rate at which a performance measure converges to the steady state under different scheduling or routing policies. For the multiclass "V" network, Gamarnik and Stolyar [20] prove the tightness of the stationary distributions of the diffusion-scaled state processes under any work conserving scheduling policy, provided that there is $\sqrt{n}$ safety staffing ( $n$ is the scaling parameter). They show that the diffusionscaled queueing processes are ergodic under all work conserving scheduling policies, and have exhibited exponential tail bounds for the stationary distribution. The proofs of these significant results utilize some natural test functions based on the total workload, but there is no uniform Foster-Lyapunov equation to exhibit the rate of convergence to the stationary distribution. For the limiting diffusion of the "V" network, when the control equals $(0, \ldots, 0,1)^{\top}$, which arises as the limit of a static priority policy, the ergodic properties established in Dieker and Gao [17] for a class of piecewise Ornstein-Uhlenbeck $(\mathrm{O}-\mathrm{U})$ processes arising in many-server queues with phase-type service times can be applied. Exponential ergodicity is also established for the limiting diffusion 
(as a special case of a more general class of SDEs) under any constant control in Arapostathis et al. [9].

The following important open questions are addressed in this paper:

(1) Is the limiting controlled diffusion exponentially ergodic under all stationary Markov controls? How different are the tail asymptotics of the invariant measures with or without abandonment?

(2) Is there a unified approach based on Foster-Lyapunov theory that can be used to establish uniform exponential ergodicity for both the limiting diffusion and the diffusion-scaled queueing processes?

We provide affirmative answers to all these questions. We consider multiclass models with (delayed) renewal arrivals, class-dependent exponential service times, and class-dependent exponential patience times. We assume that the system is operating under work-conserving and preemptive scheduling policies. It is well known that the diffusion-scaled queueing processes under such scheduling policies converge weakly to a limiting diffusion with a drift given in (2.2) and a diagonal constant covariance matrix (see $[10,26]$ ).

We start with the limiting controlled diffusion. When the controls are constant, the limiting diffusion has a piecewise linear drift and belongs to a class of piecewise O-U processes. Applying [17, Theorem 3] to our model with positive abandonment rate, one can deduce that the limiting diffusion is exponentially ergodic under a specific constant control corresponding to a static priority scheduling policy (see Remark 2.1). On the other hand, it is shown in [9, Theorem 3.5] that the limiting diffusion is exponentially ergodic under any constant control (see Remark 2.2). The proofs of these results rely on the construction of a common quadratic-type Lyapunov function for the piecewise linear equations. However, this methodology only works for constant controls, and leaves the question of stability over Markov controls open.

We exploit Lyapunov functions that are constructed in an intricate manner in order to capture both the total workload on the positive half-space and the idleness on the negative half-space. Such functions are of course quite natural, and have been used as test functions in [20] to derive tail bounds. However, for the diffusion, the total workload and idleness need to be treated with the proper "weights" or "tilting", interacting with a "smoothing" cut-off function which needs to be deployed. Such delicate care is not only needed for the drift as usual, but more importantly, for the second-order derivatives. For multiclass queueing models in the Halfin-Whitt regime, such constructions appear to be necessary in order to deal with both the workload and idleness processes simultaneously. This constitutes our first main methodology contribution in this paper.

We present Foster-Lyapunov equations that are uniform over all Markov controls, and show that

(a) if the spare capacity parameter (safety staffing) is positive, then the limiting diffusion is uniformly exponentially ergodic, and the corresponding invariant probability measures have uniform exponential tails;

(b) when the abandonment rates are all positive, regardless the spare capacity parameter being positive or negative, in addition to uniform exponential ergodicity, we show that the invariant probability measures have sub-Gaussian tails.

These answer the questions in (1) above.

We then show that the Foster-Lyapunov equations for the limiting diffusion offer a very natural tool with which we establish uniform ergodic properties for the diffusion-scaled queueing processes. This answers the question in (2). In this manner we provide a unified approach to the study of the limiting diffusion and the corresponding diffusion-scaled processes.

In the case of Poisson arrivals, by employing the same Lyapunov functions used for the limiting diffusion, we show that the corresponding results in (a) above hold for the diffusion-scaled queueing processes (see also Section 2.3). Sub-Gaussian tails are not possible for the invariant distribution of the diffusion-scaled queueing processes, and one can only hope for tails that decay faster than 
any exponential. On the other hand, when the abandonment rates are all positive, we improve somewhat on the results in [20], although a conjecture stated in that paper still remains open. Even though in the cases of Poisson and renewal arrivals the limiting diffusions agree, with the only differences lying in the covariance functions, for the analysis of the prelimit processes, we need to augment the state process in the renewal case.

With renewal arrivals, we consider the Markov process composed of the diffusion-scaled queueing processes and the interarrival age processes of the renewal arrivals. The Lyapunov functions used for the limiting diffusion are adapted to construct appropriate Lyapunov functions for the joint processes. On the other hand, the hazard rate functions and mean residual lifetime functions of the interarrival times must be also used in a proper manner to take into account the age processes as suggested in [28]. We prove the following results under the assumption that the residual lifetime function is bounded: $\left(a^{\prime}\right)$ if the spare capacity parameter (safety staffing) is positive, we prove a Foster-Lyapunov equation, which shows that the joint Markov process is positive Harris recurrent under any work-conserving stationary Markov scheduling policy; $\left(b^{\prime}\right)$ if the abandonment rates are all positive, we obtain a Foster-Lyapunov equation which shows that the first absolute moments of the invariant distribution are uniformly bounded. If we impose the additional assumption that the hazard rate function is bounded, we show that the marginal of the stationary distribution corresponding to the queueing state has exponential moments.

This work also relates to the vast literature on the validity of diffusion approximations for queues in heavy traffic. We focus on the literature of many-server queueing models in the $\mathrm{H}-\mathrm{W}$ regime, and refer the readers to $[21,14,27,23,37,38,13]$ and references therein for results in the conventional (single-server) heavy-traffic regime. For the single-class $G I / M / n$ queues, Halfin and Whitt [25] established the interchange of limits, and they used a bounding argument via single-server queues to show the tightness of the steady-state distributions of the diffusion-scaled processes. Dai et al. [15] studied the validity of the multidimensional diffusion approximations for $G I / P h / n+M$ queues with phase-type service times. Aghajani and Ramanan [1] proved the convergence of the stationary distributions of suitably scaled infinite-dimensional measure-valued processes for the $G I / G I / N$ queues in the $\mathrm{H}-\mathrm{W}$ regime, and they also studied the ergodic properties of the SPDE limit of the same model in [2]. We also refer the readers to the steady state analysis of many-server queues in $[18,19,11,12]$. All these studies are on the single-class many-server queues. For multiclass many-server queues in the $\mathrm{H}-\mathrm{W}$ regime this topic still remains wide open. The only known result is for the Markovian ' $\mathrm{N}$ ' network [33] where Stolyar proves the interchange of limits for the model without abandonment under a particular static priority policy.

Uniform exponential ergodicity can substantially simplify the study of ergodic control problems, since there is a rich body of existing theory that can be applied [4, Chapter 3]. On the other hand, if the system is not endowed with such blanket stability properties, and the running cost functional is not near-monotone, then the analysis of these problems can be quite involved. In the study of ergodic control of the "V" network in [3], a key structural property of the system dynamics had to be identified due to the lack of uniform stability and near-monotonicity of the running cost. It was assumed that all the abandonment rates are strictly positive but no positive safety staffing requirement was imposed. The results in this paper enable the study of ergodic control problems for the "V" network when there is no abandonment but there is positive safety staffing. Uniform stability properties are yet to be explored for multiclass multi-pool networks. Without such blanket stability properties, ergodic control problems for multiclass multi-pool networks have been recently studied in $[6,7,8]$, under the hypothesis that at least one abandonment rate is positive.

1.1. Notation. We summarize some of the notation used throughout the paper. We use $\mathbb{R}^{m}$ (and $\mathbb{R}_{+}^{m}$ ), $m \geq 1$, to denote real-valued $m$-dimensional (nonnegative) vectors, and write $\mathbb{R}$ for $m=1$. For $x, y \in \mathbb{R}$, we let $x \vee y=\max \{x, y\}, x \wedge y=\min \{x, y\}, x^{+}=\max \{x, 0\}$ and $x^{-}=\max \{-x, 0\}$. 
For a set $A \subseteq \mathbb{R}^{m}$, we use $A^{c}, \partial A$, and $\mathbf{1}_{A}$ to denote the complement, the boundary, and the indicator function of $A$, respectively. A ball of radius $r>0$ in $\mathbb{R}^{m}$ around a point $x$ is denoted by $\mathcal{B}_{r}(x)$, or simply as $\mathcal{B}_{r}$ if $x=0$. We also let $\mathcal{B} \equiv \mathcal{B}_{1}$. The Euclidean norm on $\mathbb{R}^{m}$ is denoted by $|\cdot|$, and $\langle\cdot, \cdot\rangle$ stands for the inner product. Also for $x \in \mathbb{R}^{m}$, we let $\|x\|_{1}:=\sum_{i}\left|x_{i}\right|, x_{\max }:=\max _{i} x_{i}$, and $x_{\min }:=\min _{i} x_{i}$, and $x^{ \pm}:=\left(x_{1}^{ \pm}, \ldots, x_{m}^{ \pm}\right)$. For a finite signed measure $\nu$ on $\mathbb{R}^{m}$, and a Borel measurable $f: \mathbb{R}^{m} \rightarrow[1, \infty)$, we define the $f$-norm of $\nu$ by

$$
\|\nu\|_{f}:=\sup _{g \in \mathcal{B}\left(\mathbb{R}^{m}\right),|g| \leq f}\left|\int_{\mathbb{R}^{m}} g(x) \nu(\mathrm{d} x)\right|,
$$

where $\mathcal{B}\left(\mathbb{R}^{m}\right)$ denotes the class of Borel measurable functions on $\mathbb{R}^{m}$. Observe that $\|\cdot\|_{1}=\|\cdot\|_{\mathrm{TV}}$, the latter denoting the total variation norm.

2. Uniform exponential ergodicity of the diffusion limit. In Section 2.1 we describe the limiting diffusion, and proceed with a summary of the results and the technical approach in Sections 2.2 and 2.3, respectively. Some important definitions are in Section 2.4, followed by the main technical results in Sections 2.5 and 2.6.

2.1. The limiting controlled diffusion. We consider a controlled $m$-dimensional stochastic differential equation (SDE) of the form

$$
\mathrm{d} X_{t}=b\left(X_{t}, U_{t}\right) \mathrm{d} t+\sigma(X(t)) \mathrm{d} W_{t}, \quad X(0)=x_{0} \in \mathbb{R}^{m},
$$

with $b: \mathbb{R}^{m} \rightarrow \mathbb{R}^{m}$ given by

$$
b(x, u)=\ell-M\left(x-\langle e, x\rangle^{+} u\right)-\langle e, x\rangle^{+} \Gamma u= \begin{cases}\ell-\left(M+(\Gamma-M) u e^{\top}\right) x, & \langle e, x\rangle>0, \\ \ell-M x, & \langle e, x\rangle \leq 0 .\end{cases}
$$

Here, $\ell \in \mathbb{R}^{m}, u \in \mathbb{R}_{+}^{m}$ satisfies $\langle e, u\rangle=1$ with $e=(1, \ldots, 1)^{\top} \in \mathbb{R}^{m}, M=\operatorname{diag}\left(\mu_{1}, \ldots, \mu_{m}\right)$ is a positive diagonal matrix, and $\Gamma=\operatorname{diag}\left(\gamma_{1}, \ldots, \gamma_{m}\right)$ with $\gamma_{i} \in \mathbb{R}_{+}, i \in \mathcal{I}:=\{1, \ldots, m\}$. The process $W_{t}$ is a standard $m$-dimensional Brownian motion, and the covariance function $\sigma: \mathbb{R}^{m} \rightarrow \mathbb{R}^{m \times m}$ is a positive diagonal matrix. Such a process arises as a limit of the suitably scaled queueing processes of multiclass Markovian many-server queues in the $\mathrm{H}-\mathrm{W}$ regime [10, 26].

In these models, if the scheduling policy is based on a static priority assignment on the queues, then the vector $u$ in (2.1) corresponds to a constant control which is an extreme point of the convex set

$$
\Delta:=\left\{u \in \mathbb{R}^{m}: u \geq 0,\langle e, u\rangle=1\right\} .
$$

REMARK 2.1. As mentioned earlier, ergodicity and exponential ergodicity of a class of piecewise $\mathrm{O}-\mathrm{U}$ processes as in (2.1) have been addressed in [17]. In this model, they assume that $M$ is a nonsingular M-matrix such that the vector $e^{\top} M$ has nonnegative components, $\Gamma=\alpha I$, and $\ell=-\beta u$ for positive constants $\alpha, \beta$, and a constant vector $u \in \Delta$. Applying their results to the multiclass $M / M / n+M$ model with abandonment, exponential ergodicity of the limiting diffusion under the specific constant control $\bar{u}=(0, \ldots, 0,1)^{\top}$, corresponding to class $m$ being given the least priority, is established in [17, Theorem 3]. On the other hand, for the multiclass $M / M / n$ model without abandonment, that is, $\Gamma=0$, positive recurrence is established for the limiting diffusion with the control $\bar{u}$ but the rate of convergence is not identified [17, Theorem 2].

REMARK 2.2. The model in (2.1) with $M$ a nonsingular M-matrix, and for constant control $U_{t}$ has also been studied extensively in [9] (as a special class of the Lévy-driven SDEs studied there). It is shown in that paper that when $\Gamma=0$, the quantity

$$
\varrho:=-\left\langle e, M^{-1} \ell\right\rangle
$$


plays a fundamental role in the characterization of stability. Specifically, it is shown in [9, Theorem 3.2] that if $\varrho>0$, then $X_{t}$ is positive recurrent under any constant control $U_{t}$, and if $\varrho<0$ $(\varrho=0)$, then it is transient (cannot be positive recurrent) under any stationary Markov control satisfying $\Gamma v(x)=0$ a.e. [9, Theorem 3.3]. Another interesting property of $\varrho$ which we find in $[9$, Corollary 5.1] is that, provided $\Gamma=0$, and the diffusion under some stationary Markov control $v$ is positive recurrent with invariant probability measure $\pi_{v}$, then necessarily

$$
\varrho=\int_{\mathbb{R}^{m}}\langle e, x\rangle^{-} \pi_{v}(\mathrm{~d} x) .
$$

This can be interpreted as follows: the 'average idleness' in the steady state always equals the spare capacity parameter. These results of course apply to the problem at hand since $M$ is a diagonal matrix. In addition, the rate of convergence is shown to be exponential if either $\Gamma u=0$ or $\Gamma u \neq 0$ for any constant control $u \in \Delta$ [9, Corollary 4.2].

Let $\mathfrak{U}_{\mathrm{sm}}$ denote the class of Borel measurable maps $v: \mathbb{R}^{m} \rightarrow \Delta$. Every element $v$ of $\mathfrak{U}_{\mathrm{sm}}$ is identified with the stationary Markov controls $U_{t}=v\left(X_{t}\right)$. Under any such control, it is well known that (2.1) has a unique strong solution which is a strong Feller process [24]. Let $P_{t}^{v}(x, \mathrm{~d} y)$ denote its transition probability.

The diffusion in (2.1) is called uniformly stable (in the sense of [4, Definition 3.3.3]), if under any $v \in \mathfrak{U}_{\mathrm{sm}}$, the process $X_{t}$ is positive recurrent and the collection of invariant probability measures is tight. We say that (2.1) is uniformly exponentially ergodic, if it is uniformly stable and there exist positive constants $C$ and $\gamma$ and a function $\mathcal{V}: \mathbb{R}^{m} \rightarrow[1, \infty)$ such that

$$
\left\|P_{t}^{v}(x, \cdot)-\pi_{v}(\cdot)\right\|_{\mathrm{TV}} \leq C \mathcal{V}(x) \mathrm{e}^{-\gamma t} \quad \forall(x, t) \in \mathbb{R}^{m} \times \mathbb{R}_{+},
$$

and all $v \in \mathfrak{U}_{\mathrm{sm}}$.

2.2. Brief summary of the results. In Theorem 2.1 we show that if $\varrho>0$, then $(2.1)$ is uniformly exponentially ergodic. Therefore, when $\Gamma=0,(2.4)$ holds over all stationary Markov controls $v \in \mathfrak{U}_{\mathrm{sm}}$. In addition, the invariant probability measures have uniform exponential tails, and by that, we mean that there exists some $\varepsilon>0$, such that $\sup _{v \in \mathfrak{U}_{\text {sm }}} \int_{\mathbb{R}^{m}} \mathrm{e}^{\varepsilon|x|} \pi_{v}(\mathrm{~d} x)<\infty$. On the other hand, if $\Gamma>0$, then the associated invariant probability measures have sub-Gaussian tails, that is, $\sup _{v \in \mathfrak{U}_{\mathrm{sm}}} \int_{\mathbb{R}^{m}} \mathrm{e}^{\varepsilon|x|^{2}} \pi(\mathrm{d} x)<\infty$ for some $\varepsilon>0$ (see Theorem 2.2).

In Section 3 we address the $n$-server networks. We first present the results for the models with (delayed) renewal arrival processes in Section 3.2. The counterpart of Theorem 2.1 here is given in Theorem 3.1, and this is established for renewal arrivals (this should be compared to [20, Theorem 2]). In this theorem, the hazard rate functions are assumed bounded. This is a rather strong assumption, but the result, which asserts uniform exponential tails for the invariant distributions under work-conserving stationary Markov policies is equally strong. With strictly positive abandonment parameters, and with the hazard rate function only locally bounded, we establish uniform stability of the queueing system under all work-conserving stationary Markov policies in Theorem 3.2. With possibly zero abandonment in all classes, and with positive $\sqrt{n}$ safety staffing, we show in Theorem 3.3, that the combined renewal age and queueing state process is positive Harris recurrent. In addition, if the limit of the safety staffing is positive, the invariant probability distributions are tight. In this result, the hazard rate function is assumed only locally bounded.

Networks with Poisson arrivals are studied in Section 3.3. We show in Corollary 3.1 that positive spare capacity implies exponential ergodicity. However, as noted in [20] the invariant distribution of an $n$-server network cannot have a sub-Gaussian tail. This property is recovered only at the weak limit as $n \rightarrow \infty$, and it is worthwhile comparing [20, Theorem 4] with Theorem 2.2, which in addition shows uniform exponential ergodicity. When all abandonment rates are positive, we 
can only exhibit a stronger Foster-Lyapunov equation (see Theorem 3.4) which implies that $\mathrm{e}^{\delta|x|}$ is uniformly integrable over the invariant probability distributions for any $\delta>0$.

In addition to these results, we investigate the properties in Theorem 2 (i) and Theorem 4 (i) of [20]. We provide proofs of the analogous results for the limiting diffusion in Lemma 2.2 and Theorem 2.3, respectively, using Foster-Lyapunov techniques. The counterpart of Lemma 2.2 for the $n$-system is given in Theorem 3.5 and is an improvement over the statement in [20, Theorem 2 (i)]. However, we have not been able to prove or disprove the related conjecture in [20, p. 33].

2.3. Summary of the technical approach. The first important step in the study of this problem is the construction of appropriate Lyapunov functions. We use two building blocks for these functions: one represents the total workload, and the other is a measure of idleness. The scaling of these in (2.9) plays a crucial role. Two scaling parameters are used: $\theta$ to balance the mix of workload and idleness, and $\varepsilon$ to handle the terms arising from the second derivatives in the extended generator of the controlled diffusion. Equally important are the cones in Definition 2.1. Note that although the drift of the diffusion is piecewise-linear when the control is constant, it becomes quite complicated under a (stationary) Markov control. Careful analysis of the drift of the diffusion in (2.2) on these cones enables us to obtain the drift inequalities and Foster-Lyapunov equations in Lemma 2.1 and Theorems 2.1 and 2.2. The more specialized results in Section 2.6 involve Lyapunov functions which are sums of two exponentials.

The relation between the prelimit dynamics and the limiting diffusion can be described as follows. For a model with Poisson arrivals, the process $\left\{\hat{X}^{n}(t)\right\}_{t \geq 0}$ describing the (diffusion-scaled) total number of jobs in the system is a controlled Markov process with generator (see (3.12) and (3.37))

$$
\widehat{\mathcal{A}}_{z}^{n} f(\hat{x}):=\sum_{i \in \mathcal{I}} \lambda_{i}^{n}\left(f\left(\hat{x}+n^{-1 / 2} e_{i}\right)-f(\hat{x})\right)+\sum_{i \in \mathcal{I}}\left(\mu_{i}^{n} z_{i}+\gamma_{i}^{n} q_{i}(\hat{x}, z)\right)\left(f\left(\hat{x}-n^{-1 / 2} e_{i}\right)-f(\hat{x})\right) .
$$

Here, the vector $z=\left(z_{1}, \ldots, z_{m}\right) \in \mathbb{Z}_{+}^{m}$ is the control parameter, with $z_{i}$ denoting the number of jobs of class $i$ in service, $\left\{\lambda_{i}^{n}\right\}_{i \in \mathcal{I}},\left\{\mu_{i}^{n}\right\}_{i \in \mathcal{I}}$, and $\left\{\gamma_{i}^{n}\right\}_{i \in \mathcal{I}}$ are the arrival, service rates, and abandonment rates, respectively, and $q=\left(q_{1}, \ldots, q_{m}\right)$ is the vector of queue sizes. Using the diffusion-scaled variables $\hat{z}^{n}$ and $\hat{q}^{n}$ defined in (3.7) as

$$
\hat{z}_{i}^{n}:=\frac{1}{\sqrt{n}}\left(z_{i}-\frac{\lambda_{i}^{n}}{\mu_{i}^{n}}\right)-\frac{\varrho^{n}}{m}, \quad \text { and } \quad \hat{q}_{i}^{n}:=\frac{q_{i}(x, z)}{\sqrt{n}},
$$

we obtain

$$
\begin{aligned}
\widehat{\mathcal{A}}_{z}^{n} f(\hat{x})=\sum_{i \in \mathcal{I}} \frac{\lambda_{i}^{n}}{n} \frac{f\left(\hat{x}+n^{-1 / 2} e_{i}\right)-2 f(\hat{x})+f\left(\hat{x}-n^{-1 / 2} e_{i}\right)}{n^{-1}} & \\
& +\sum_{i \in \mathcal{I}}\left(\mu_{i}^{n} \frac{\varrho^{n}}{m}+\mu_{i}^{n} \hat{z}_{i}^{n}+\gamma_{i}^{n} \hat{q}_{i}^{n}\right) \frac{f\left(\hat{x}-n^{-1 / 2} e_{i}\right)-f(\hat{x})}{n^{-1 / 2}} .
\end{aligned}
$$

As shown in (3.32), for any work-conserving job allocation $z \in \mathbb{Z}_{+}^{m}$, there exists a vector $u \in \Delta$ such that $\hat{z}^{n}=\hat{x}-\langle e, \hat{x}\rangle^{+} u$, and $\hat{q}^{n}=\langle e, \hat{x}\rangle^{+} u$. Using these identities in (2.5), and letting $n \rightarrow \infty$, we obtain the generator of the controlled diffusion in (2.1) (see also Remark 2.3). There is some difficulty though with translating the Foster-Lyapunov equation for the diffusion into an analogous equation for the operator $\widehat{\mathcal{A}}_{z}^{n}$. This is because, whereas $\hat{z}^{n}$ is of order $\sqrt{n}$, the queue sizes $\hat{q}^{n}$ are not bounded. We circumvent this problem by establishing drift inequalities in Lemma 2.1 for the truncated drift given in (2.14). This facilitates using the same Lyapunov function for the stability analysis of the diffusion and the prelimit, and consequently, we have a unified approach to the problem.

When studying the diffusion-scaled model with renewal arrivals, the Lyapunov function has to be augmented to account for the age processes (see (3.8)). The analysis is more intricate in this case, and deriving the Foster-Lyapunov equations in Theorem 3.1 requires extra care. 
REMARK 2.3. Note that if we let $\zeta=\frac{\varrho}{m} e+M^{-1} \ell$, with $\varrho$ as in (2.3), then a mere translation of the origin of the form $\tilde{X}_{t}=X_{t}-\zeta$ results in a diffusion of the form (2.1) with the constant $\ell$ in the drift taking the form $\ell=-\frac{\rho}{m} M e$. Therefore, without loss of generality, we assume throughout the paper that the drift in (2.2) takes the form

$$
b(x, u)=-\frac{\varrho}{m} M e-M\left(x-\langle e, x\rangle^{+} u\right)-\langle e, x\rangle^{+} \Gamma u .
$$

For $f \in \mathcal{C}^{2}\left(\mathbb{R}^{m}\right)$ and $u \in \Delta$, we define $a(x)=\left(a^{i j}(x)\right)_{1 \leq i, j \leq m}:=\sigma(x) \sigma(x)^{\top}$, and

$$
\mathcal{L}_{u} f(x)=\frac{1}{2} \operatorname{trace}\left(a(x) \nabla^{2} f(x)\right)+\langle b(x, u), \nabla f(x)\rangle,
$$

with $\nabla^{2} f$ denoting the Hessian of $f$.

2.4. Preliminaries. We start with two definitions.

Definition 2.1. For $\delta \in[0,1]$, we define the following cones

$$
\begin{aligned}
& \mathcal{K}_{\delta}^{+}:=\left\{x \in \mathbb{R}^{m}:\langle e, x\rangle \geq \delta\|x\|_{1}\right\}, \\
& \mathcal{K}_{\delta}^{-}:=\left\{x \in \mathbb{R}^{m}:\langle e, x\rangle \leq-\delta\|x\|_{1}\right\} .
\end{aligned}
$$

Note that $\mathcal{K}_{0}^{+}\left(\mathcal{K}_{0}^{-}\right)$is the nonnegative (nonpositive) canonical half-space, and $\mathcal{K}_{1}^{+}\left(\mathcal{K}_{1}^{-}\right)$is the nonnegative (nonpositive) closed orthant. Also we have the following identities:

$$
\left\langle e, x^{+}\right\rangle=\frac{1 \pm \delta}{2}\|x\|_{1}, \quad\left\langle e, x^{-}\right\rangle=\frac{1 \mp \delta}{2}\|x\|_{1} \quad \text { for } \quad x \in \partial \mathcal{K}_{\delta}^{ \pm}, \delta \in[0,1] .
$$

We fix some convex function $\psi \in \mathcal{C}^{2}(\mathbb{R})$ with the property that $\psi(t)$ is constant for $t \leq-1$, and $\psi(t)=t$ for $t \geq 0$. This is defined by

$$
\psi(t):= \begin{cases}-\frac{1}{2}, & t \leq-1, \\ (t+1)^{3}-\frac{1}{2}(t+1)^{4}-\frac{1}{2}, & t \in[-1,0], \\ t, & t \geq 0 .\end{cases}
$$

For $\varepsilon>0$ we define

$$
\psi_{\varepsilon}(t):=\psi(\varepsilon t),
$$

Thus $\psi_{\varepsilon}(t)=\varepsilon t$ for $t \geq 0$. A simple calculation also shows that $\psi_{\varepsilon}^{\prime \prime}(t) \leq \frac{3}{2} \varepsilon^{2}$.

Definition 2.2. We let $\beta_{i}:=\frac{\gamma_{i}}{\mu_{i}}$ for $i \in \mathcal{I}$. With $\theta$ and $\varepsilon$ positive constants, we define

$$
\begin{gathered}
\Psi_{\varepsilon}(x):=\sum_{i \in \mathcal{I}} \frac{\psi_{\varepsilon}\left(x_{i}\right)}{\mu_{i}}, \quad \Psi(x):=\sum_{i \in \mathcal{I}} \frac{\psi\left(x_{i}\right)}{\mu_{i}}, \\
\text { and } \Psi_{\varepsilon, \theta}^{*}(x):=\varepsilon \theta \Psi(-x)+\Psi_{\varepsilon}(x) .
\end{gathered}
$$

The function $\Psi$ plays a fundamental role in our analysis. The quantity $\Psi\left(x^{+}\right)$represents of course the total workload, while $\Psi\left(x^{-}\right)$is a measure of idleness. These functions, without the smooth cutoff part, are also used in [20] as test functions to estimate the tails of the invariant distribution of the prelimit diffusion-scaled processes.

The function $\Psi_{\varepsilon, \theta}^{*}$ "follows" the norm $\|\cdot\|_{1}$, in the sense that

$$
\varepsilon \frac{1 \wedge \theta}{\mu_{\max }}\|x\|_{1}-\frac{m}{2} \leq \Psi_{\varepsilon, \theta}^{*}(x) \leq \varepsilon \frac{1 \vee \theta}{\mu_{\min }}\|x\|_{1} .
$$


We also have $\psi^{\prime}(-1 / 2)=1 / 2$, from which we obtain

$$
\sum_{i \in \mathcal{I}} \psi_{\varepsilon}^{\prime}\left(x_{i}\right) x_{i} \geq \varepsilon\left\|x^{+}\right\|_{1}-\frac{m}{2}, \quad \text { and }-\sum_{i \in \mathcal{I}} \psi^{\prime}\left(-x_{i}\right) x_{i} \geq\left\|x^{-}\right\|_{1}-\frac{m}{2} .
$$

Note also that

$$
-\varepsilon \sum_{i \in \mathcal{I}} \psi^{\prime}\left(-x_{i}\right) x_{i} \leq \varepsilon\langle e, x\rangle \leq \sum_{i \in \mathcal{I}} \psi_{\varepsilon}^{\prime}\left(x_{i}\right) x_{i}
$$

Using the parameter $\beta_{i}$ in Definition 2.2 and (2.6), we write the following identities, which we use frequently in the rest of the paper.

$$
\begin{gathered}
\left\langle\nabla \Psi_{\varepsilon}(x), b(x, u)\right\rangle=-\frac{\varrho}{m} \sum_{i \in \mathcal{I}} \psi_{\varepsilon}^{\prime}\left(x_{i}\right)-\sum_{i \in \mathcal{I}} \psi_{\varepsilon}^{\prime}\left(x_{i}\right) x_{i}+\langle e, x\rangle^{+} \sum_{i \in \mathcal{I}} \psi_{\varepsilon}^{\prime}\left(x_{i}\right)\left(1-\beta_{i}\right)^{+} u_{i} \\
-\langle e, x\rangle^{+} \sum_{i \in \mathcal{I}} \psi_{\varepsilon}^{\prime}\left(x_{i}\right)\left(\beta_{i}-1\right)^{+} u_{i}, \\
\langle\nabla \Psi(-x), b(x, u)\rangle=\frac{\varrho}{m} \sum_{i \in \mathcal{I}} \psi^{\prime}\left(-x_{i}\right)+\sum_{i \in \mathcal{I}} \psi^{\prime}\left(-x_{i}\right) x_{i}-\langle e, x\rangle^{+} \sum_{i \in \mathcal{I}} \psi^{\prime}\left(-x_{i}\right)\left(1-\beta_{i}\right)^{+} u_{i} \\
+\langle e, x\rangle^{+} \sum_{i \in \mathcal{I}} \psi^{\prime}\left(-x_{i}\right)\left(\beta_{i}-1\right)^{+} u_{i} .
\end{gathered}
$$

2.5. Main results on uniform exponential ergodicity. The following lemma presents some important drift inequalities which are used frequently throughout the paper. Recall the definitions in (2.9). In order to apply the drift inequalities for the diffusion to the prelimit in Section 3, we often need to truncate the diffusion-scaled queueing processes. To prepare for this, we present a more general version of these inequalities than what is needed in this section.

For a constant $c \in[1, \infty]$ we define $b_{c}(x, u):=\left(b_{c}^{1}(x, u), \ldots, b_{c}^{m}(x, u)\right)^{\top}$, with

$$
b_{c}^{i}(x, u):=-\frac{\varrho \mu_{i}}{m}-\mu_{i}\left(x_{i}-\langle e, x\rangle^{+} u_{i}\right)-\gamma_{i}\langle e, x\rangle^{+} u_{i} \mathbf{1}_{\left\{x_{i} \leq c\right\}} .
$$

Lemma 2.1. Assume $\varrho>0$, and let $\theta$ be a positive constant satisfying

$$
\theta\left(\beta_{\max }-1\right)^{+} \leq 1
$$

Then, the function

$$
V(x):=\exp \left(\Psi_{\epsilon, \theta}^{*}(x)\right)=\exp \left(\varepsilon \theta \Psi(-x)+\Psi_{\varepsilon}(x)\right)
$$

satisfies, for any constant $c \in[1, \infty]$,

$$
\left\langle\nabla V(x), b_{c}(x, u)\right\rangle \leq \begin{cases}\varepsilon\left(\theta \varrho+\frac{m}{2 \varepsilon}(1+\varepsilon \theta)-(\theta \wedge 1)\|x\|_{1}\right) V(x) & \forall x \in \mathcal{K}_{0}^{-}, \\ -\varepsilon\left(\frac{\varrho}{m}-\theta \varrho-\theta \frac{m}{2}+\theta\left\|x^{-}\right\|_{1}\right) V(x) & \forall(x, u) \in \mathcal{K}_{0}^{+} \times \Delta .\end{cases}
$$

Proof. The bound on $\mathcal{K}_{0}^{-}$follows by first multiplying $(2.13 \mathrm{~b})$ by $\varepsilon \theta$, then adding this equation to $(2.13 \mathrm{a})$, and using $(2.11)$.

We proceed to derive the stated bound on $\mathcal{K}_{0}^{+} \times \Delta$. Note that

$$
\left\langle\nabla \Psi(-x), b_{c}(x, u)\right\rangle=\langle\nabla \Psi(-x), b(x, u)\rangle,
$$

that is, it is equal to the right-hand side of (2.13b) for any $c \geq 1$, since $\psi^{\prime}(-r)=0$ for $r \geq 1$. We write

$$
\begin{aligned}
\left\langle\nabla \Psi_{\varepsilon}(x), b_{c}(x, u)\right\rangle=-\frac{\varrho}{m} \sum_{i \in \mathcal{I}} \psi_{\varepsilon}^{\prime}\left(x_{i}\right)-\sum_{i \in \mathcal{I}} \psi_{\varepsilon}^{\prime}\left(x_{i}\right) & \left(x_{i}-\langle e, x\rangle^{+} u_{i}\right) \\
& -\langle e, x\rangle^{+} \sum_{i \in \mathcal{I}} \psi_{\varepsilon}^{\prime}\left(x_{i}\right) \beta_{i} u_{i} \mathbf{1}_{\left\{x_{i} \leq c\right\}} .
\end{aligned}
$$


It holds that

$$
-\frac{\varrho}{m} \sum_{i \in \mathcal{I}} \psi_{\varepsilon}^{\prime}\left(x_{i}\right) \leq-\varepsilon \frac{\varrho}{m} \quad \text { on } \mathcal{K}_{0}^{+}
$$

Also, by (2.11), we have

$$
\theta \varepsilon \frac{\varrho}{m} \sum_{i \in \mathcal{I}} \psi^{\prime}\left(-x_{i}\right)+\theta \varepsilon \sum_{i \in \mathcal{I}} \psi^{\prime}\left(-x_{i}\right) x_{i} \leq \varepsilon \theta \varrho+\varepsilon \theta \frac{m}{2}-\varepsilon \theta\left\|x^{-}\right\|_{1} \quad \text { on } \mathbb{R}^{m},
$$

and

$$
\sum_{i \in \mathcal{I}} \psi_{\varepsilon}^{\prime}\left(x_{i}\right)\left(x_{i}-\langle e, x\rangle^{+} u_{i}\right) \geq 0 \quad \text { for } x \in \mathcal{K}_{0}^{+}
$$

by (2.12). Thus, if $\beta_{\max } \leq 1$, then it is clear from (2.13a) and (2.18)-(2.21), that (2.17) holds for any positive $\varepsilon$ and $\theta$.

Next suppose $\beta_{\max }>1$. We proceed by carefully comparing the terms in $(2.13 \mathrm{~b})$ and $(2.18)$. Define

$$
\widehat{\mathcal{I}}:=\left\{i \in \mathcal{I}: \gamma_{i}>\mu_{i}\right\}, \quad \widehat{\mathcal{I}}_{+}(x):=\left\{i \in \widehat{\mathcal{I}}: x_{i} \geq 0\right\}, \quad \text { and } \widehat{\mathcal{I}}_{-}(x):=\left\{i \in \widehat{\mathcal{I}}: x_{i}<0\right\} .
$$

Since $\theta\left(\beta_{\max }-1\right)^{+} \leq 1$, and $\beta_{i}>1$ on $\widehat{\mathcal{I}}$, we have

$$
\varepsilon \theta \sum_{i \in \widehat{\mathcal{I}}_{+}(x)} \psi^{\prime}\left(-x_{i}\right)\left(\beta_{i}-1\right)^{+} u_{i} \leq \sum_{i \in \widehat{\mathcal{I}}_{+}(x)} \psi_{\varepsilon}^{\prime}\left(x_{i}\right) \beta_{i} u_{i} \mathbf{1}_{\left\{x_{i} \leq c\right\}}
$$

which we combine with

$$
\sum_{i \in \widehat{\mathcal{I}}_{-}(x)} \psi_{\varepsilon}^{\prime}\left(x_{i}\right) u_{i}-\sum_{i \in \widehat{\mathcal{I}}_{-}(x)} \psi_{\varepsilon}^{\prime}\left(x_{i}\right) \beta_{i} u_{i} \mathbf{1}_{\left\{x_{i} \leq c\right\}} \leq 0
$$

to write

$$
\varepsilon \theta \sum_{i \in \widehat{\mathcal{I}}_{+}(x)} \psi^{\prime}\left(-x_{i}\right)\left(\beta_{i}-1\right)^{+} u_{i}+\sum_{i \in \widehat{\mathcal{I}}_{-}(x)} \psi_{\varepsilon}^{\prime}\left(x_{i}\right) u_{i}-\sum_{i \in \widehat{\mathcal{I}}} \psi_{\varepsilon}^{\prime}\left(x_{i}\right) \beta_{i} u_{i} \mathbf{1}_{\left\{x_{i} \leq c\right\}} \leq 0 .
$$

By the definitions in (2.22), we have the identity

$$
\varepsilon \sum_{i \in \widehat{\mathcal{I}}_{-}(x)} \psi^{\prime}\left(-x_{i}\right) u_{i}=\varepsilon \sum_{i \in \hat{\mathcal{I}}} u_{i}-\sum_{i \in \widehat{\mathcal{I}}_{+}(x)} \psi_{\varepsilon}^{\prime}\left(x_{i}\right) u_{i} .
$$

Using again the fact that $\psi^{\prime}(-r)=0$ for $r \geq 1$, we obtain

$$
\begin{aligned}
\varepsilon \theta\langle e, x\rangle^{+} \sum_{i \in \widehat{\mathcal{I}}_{-}(x)} \psi^{\prime}\left(-x_{i}\right)\left(\beta_{i}-1\right)^{+} u_{i} & \leq \varepsilon \theta\left(\beta_{\max }-1\right)^{+}\langle e, x\rangle^{+} \sum_{i \in \widehat{\mathcal{I}}_{-}(x)} \psi^{\prime}\left(-x_{i}\right) u_{i} \\
& \leq \varepsilon\langle e, x\rangle^{+} \sum_{i \in \widehat{\mathcal{I}}_{-}(x)} \psi^{\prime}\left(-x_{i}\right) u_{i} \\
& \leq \varepsilon\langle e, x\rangle^{+} \sum_{i \in \widehat{\mathcal{I}}_{i}} u_{i}-\langle e, x\rangle^{+} \sum_{i \in \widehat{\mathcal{I}}_{+}(x)} \psi_{\varepsilon}^{\prime}\left(x_{i}\right) u_{i}
\end{aligned}
$$

for all $(x, u) \in \mathcal{K}_{0}^{+} \times \Delta$. In the second inequality of $(2.25)$ we used the fact that $\theta\left(\beta_{\max }-1\right)^{+} \leq 1$, and in the third we used (2.24). Multiplying (2.23) by by $\langle e, x\rangle^{+}$, and adding it to (2.25), and then combining the resulting sum with the inequality

$$
\langle e, x\rangle^{+} \sum_{i \in \widehat{\mathcal{I}}^{c}} \psi_{\varepsilon}^{\prime}\left(x_{i}\right) u_{i}-\varepsilon\langle e, x\rangle^{+} \sum_{i \in \widehat{\mathcal{I}}^{c}} u_{i} \leq 0,
$$


where $\widehat{\mathcal{I}}^{\mathrm{c}}$ denote the complement of $\widehat{\mathcal{I}}$ with respect to $\mathcal{I}$, we obtain

$$
\begin{aligned}
& \varepsilon \theta\langle e, x\rangle^{+} \sum_{i \in \widehat{\mathcal{I}}} \psi^{\prime}\left(-x_{i}\right)\left(\beta_{i}-1\right)^{+} u_{i}-\varepsilon\langle e, x\rangle^{+}+\sum_{i \in \mathcal{I}} \psi_{\varepsilon}^{\prime}\left(x_{i}\right)\langle e, x\rangle^{+} u_{i} \\
&-\langle e, x\rangle^{+} \sum_{i \in \widehat{\mathcal{I}}} \beta_{i} \psi_{\varepsilon}^{\prime}\left(x_{i}\right) u_{i} \mathbf{1}_{\left\{x_{i} \leq c\right\}} \leq 0 .
\end{aligned}
$$

Replacing the term $-\varepsilon\langle e, x\rangle^{+}$in $(2.26)$ with $-\sum_{i \in \mathcal{I}} \psi_{\varepsilon}^{\prime}\left(x_{i}\right) x_{i}$ preserves this inequality by $(2.12)$. Thus, by (2.13b), (2.18)-(2.20), and (2.26), we obtain

$$
\left\langle\nabla \Psi_{\varepsilon, \theta}^{*}(x), b_{c}(x, u)\right\rangle \leq-\varepsilon \frac{\varrho}{m}+\varepsilon \theta \varrho+\varepsilon \theta \frac{m}{2}-\varepsilon \theta\left\|x^{-}\right\|_{1} \quad \forall(x, u) \in \mathcal{K}_{0}^{+} \times \Delta,
$$

from which the second bound in (2.17) follows. This completes the proof.

Recall the definitions in (1.1) and (2.7). Also recall that $\pi_{v}$ denotes the invariant probability measure of the process governed by (2.1) for a control $v \in \mathfrak{U}_{\mathrm{sm}}$, under which $\{X(t)\}_{t \geq 0}$ is positive recurrent.

Theorem 2.1. Assume that $\varrho>0$, and in addition to $(2.15)$, let

$$
0<\theta \leq \frac{\varrho}{3 m(2 \varrho+m)} .
$$

Then the following hold:

(a) There exists $\varepsilon_{0}>0$, such that for each $\varepsilon \leq \varepsilon_{0}$, the function $V$ in (2.16), satisfies the FosterLyapunov equation

$$
\mathcal{L}_{u} V(x) \leq \kappa_{0}-\varepsilon\left(\frac{\varrho}{2 m}+\theta\left\|x^{-}\right\|_{1}\right) V(x) \quad \forall(x, u) \in \mathbb{R}^{m} \times \Delta,
$$

for some positive constant $\kappa_{0}$ which depends only on $\varepsilon$ and $\theta$. In particular, the process $\{X(t)\}_{t \geq 0}$ is positive recurrent under any control $v \in \mathfrak{U}_{\mathrm{sm}}$, and

$$
\int_{\mathbb{R}^{m}} V(x) \pi_{v}(\mathrm{~d} x) \leq \frac{2 m}{\varepsilon \varrho} \kappa_{0} .
$$

(b) There exist positive constants $\gamma$ and $C_{\gamma}$ such that

$$
\left\|P_{t}^{v}(x, \cdot)-\pi_{v}(\cdot)\right\|_{V} \leq C_{\gamma} V(x) \mathrm{e}^{-\gamma t} \quad \forall(t, x) \in \mathbb{R}_{+} \times \mathbb{R}^{m}, \forall v \in \mathfrak{U}_{\mathrm{sm}} .
$$

Proof. Recall the definitions in (2.9), and also define

$$
\psi_{\varepsilon, \theta}^{*}(t):=\varepsilon \theta \psi(-t)+\psi_{\varepsilon}(t), \quad t \in \mathbb{R} .
$$

Write the diffusion matrix as $\sigma=\operatorname{diag}\left(2 \tilde{\lambda}_{1}, \ldots, 2 \tilde{\lambda}_{m}\right)^{1 / 2}$. For the queueing network, $\tilde{\lambda}_{i}=\frac{1}{2} \lambda_{i}\left(1+c_{a, i}^{2}\right)$, where $\lambda_{i}$ is the arrival rate (of the fluid limit), and $c_{a, i}^{2}$ is squared coefficient of variation of the renewal arrival process (see Section 3.1). In the case of a system with Poisson arrivals, $\tilde{\lambda}_{i}=\lambda_{i}$, as in (2.5). See Section 3.1 for the definition of these parameters. We have

$$
\frac{1}{2} \operatorname{trace}\left(a \nabla^{2} V(x)\right)=\left(\sum_{i \in \mathcal{I}} \frac{\tilde{\lambda}_{i}}{\mu_{i}}\left(\psi_{\varepsilon, \theta}^{*}\right)^{\prime \prime}\left(x_{i}\right)+\sum_{i \in \mathcal{I}} \frac{\tilde{\lambda}_{i}}{\mu_{i}^{2}}\left[\left(\psi_{\varepsilon, \theta}^{*}\right)^{\prime}\left(x_{i}\right)\right]^{2}\right) V(x) \quad \forall x \in \mathbb{R}^{m} .
$$

Recall that $\psi_{\varepsilon}^{\prime \prime} \leq \frac{3}{2} \varepsilon^{2}$. Therefore, since also $\psi_{\varepsilon}^{\prime} \leq \varepsilon, \theta \leq 1$, and $\sum_{i} \frac{\tilde{\lambda}_{i}}{\mu_{i}}=1$ (see (3.1)), we obtain

$$
\frac{1}{2} \operatorname{trace}\left(a \nabla^{2} V(x)\right) \leq \varepsilon\left(\frac{3}{2}(\varepsilon+\theta)+\varepsilon \bar{C}\right) V(x), \quad \text { with } \quad \bar{C}:=\sum_{i \in \mathcal{I}} \frac{\tilde{\lambda}_{i}}{\mu_{i}^{2}} .
$$


We also have $\theta \varrho+\theta \frac{m}{2} \leq \frac{\varrho}{6 m}$, and $\frac{3}{2} \theta \leq \frac{\varrho}{4 m}$ by (2.27). Thus (2.28) follows from (2.17) by selecting $\varepsilon<\frac{\varrho}{6 m(3+2 C)}$, while $(2.29)$ follows by $(2.28)$ and Itô's formula in the usual manner.

We now turn to part (b). Write $(2.28)$ as

$$
\mathcal{L}_{u} V(x) \leq \kappa_{0}-\kappa_{1} V(x),
$$

We follow the proof of $\left[29\right.$, Theorem 6.1] which uses a $\delta$-skeleton chain $\left\{X_{\delta n}\right\}_{n \in \mathbb{N}}$. Note that we can use any $\delta>0$, because $P_{\delta}^{v}(x, B)>0$ for any set $B$ with positive Lebesgue measure. Thus, for simplicity, we use $\delta=1$. Then, with $t=n+s, s \in[0,1)$, we have

$$
\begin{aligned}
\left\|P_{t}^{v}(x, \cdot)-\pi_{v}(\cdot)\right\|_{V} & =\sup _{g \in \mathcal{B}\left(\mathbb{R}^{m}\right),|g| \leq V}\left|\int_{\mathbb{R}^{m}} P_{n+s}^{v}(x, \mathrm{~d} y) g(y)-\int_{\mathbb{R}^{m}} g(y) \pi_{v}(\mathrm{~d} y)\right| \\
& \leq \int_{\mathbb{R}^{m}} P_{s}^{v}(x, \mathrm{~d} y)\left\|P_{n}^{v}(y, \cdot)-\pi_{v}(\cdot)\right\|_{V} \cdot
\end{aligned}
$$

Next, we estimate $\left\|P_{n}^{v}(y, \cdot)-\pi_{v}(\cdot)\right\|_{V}$ using [30, Theorem 2.3]. Using Itô's formula and (2.32), we obtain (see [4, Lemma 2.5.5])

$$
\int_{\mathbb{R}^{m}} P_{t}^{v}(x, \mathrm{~d} y) V(y)=\mathbb{E}_{x}^{v}\left[V\left(X_{t}\right)\right] \leq \frac{\kappa_{0}}{\kappa_{1}}+\mathrm{e}^{-\kappa_{1} t} V(x) \quad \forall(t, x) \in \mathbb{R}_{+} \times \mathbb{R}^{m}, \forall v \in \mathfrak{U}_{\mathrm{sm}} .
$$

Therefore, with $\mathcal{B}$ a ball such that $\frac{\kappa_{0}}{\kappa_{1}} \leq \mathrm{e}^{-\frac{\kappa_{1}}{2}}\left(1-\mathrm{e}^{-\frac{\kappa_{1}}{2}}\right) V(x)$ for $x \in \mathcal{B}^{c}$, we have

$$
\int_{\mathbb{R}^{m}} P_{1}^{v}(x, \mathrm{~d} y) V(y) \leq \mathrm{e}^{-\frac{\kappa_{1}}{2}} V(x)+\frac{\kappa_{0}}{\kappa_{1}} \mathbf{1}_{\mathcal{B}}(x),
$$

which establishes equation (14) in [30].

The inequality in (2.29) implies that the collection of invariant probability measures $\left\{\pi_{v}: v \in \mathfrak{U}_{\mathrm{sm}}\right\}$ is tight. By the invariance of $\pi_{v}$, tightness, and the Harnack inequality applied to the densities of $\pi_{v}$ (see [4, Lemma 3.2.4(b)]), we have

$$
\int_{\mathbb{R}^{m}} \pi_{v}(\mathrm{~d} y) P_{1 / 2}^{v}(y, \mathcal{B})=\pi_{v}(\mathcal{B}) \geq \beta_{0}>0
$$

for some constant $\beta_{0}$ independent of $v \in \mathfrak{U}_{\mathrm{sm}}$. Using tightness once more, we can select a ball $B_{R} \supset \mathcal{B}$ such that

$$
\int_{B_{R}} \pi_{v}(\mathrm{~d} y) P_{1 / 2}^{v}(y, \mathcal{B}) \geq \frac{\beta_{0}}{2} .
$$

This implies that $\sup _{y \in B_{R}} P_{1 / 2}^{v}(y, \mathcal{B}) \geq \frac{\beta_{0}}{2}$. We now employ the parabolic Harnack inequality for operators in nondivergence form [22, Theorem 4.1] (for a simpler statement which uses the notation in this paper see [5, Theorem 4.7]). The parabolic Harnack inequality asserts that there exists a positive constant $C_{\mathrm{H}}$ such that

$$
\sup _{y \in B_{R}} P_{1 / 2}^{v}(y, \mathcal{B}) \leq C_{\mathrm{H}} \inf _{y \in B_{R}} P_{1}^{v}(y, \mathcal{B}) \quad \forall v \in \mathfrak{U}_{\mathrm{sm}} .
$$

Therefore, $P_{1}^{v}(x, \mathcal{B}) \geq \frac{1}{2} C_{\mathrm{H}}^{-1} \beta_{0}$ for all $x \in \mathcal{B}$ and $v \in \mathfrak{U}_{\mathrm{sm}}$. Thus, with $\delta_{0}:=\frac{1}{4} C_{\mathrm{H}}^{-1} \beta_{0}$ we can write

$$
\eta:=\inf _{y \in \mathcal{B}} P_{1}^{v}(x, \mathcal{B})-\delta_{0} \geq \delta_{0} \quad \forall x \in \mathcal{B}, \forall v \in \mathfrak{U}_{\text {sm }},
$$

which establishes [30, equation (23)].

As seen then from equations (19)-(20) and (24)-(25) in [30, Theorem 2.3] there exist positive constants $C_{0}$ and $\gamma$ depending only on $\kappa_{0}, \kappa_{1}, \eta$, and $\delta_{0}$, such that

$$
\left\|P_{n}^{v}(x, \cdot)-\pi_{v}(\cdot)\right\|_{V} \leq C_{0} \mathrm{e}^{-\gamma n} V(x) .
$$

Thus, using (2.35) in (2.33), and applying (2.34) once more, we obtain (2.30) for a constant $C_{\gamma}$ independent of $v \in \mathfrak{U}_{\mathrm{sm}}$. This completes the proof. 
Throughout the paper we let $K_{r}$, or $K(r)$, for $r>0$, denote the closed cube

$$
K_{r}:=\left\{x \in \mathbb{R}^{m}:\|x\|_{1} \leq r\right\} .
$$

We also let $\bar{\psi}_{\varepsilon}=\psi_{\varepsilon}+1$ so that the function is strictly positive, and define $\bar{\Psi}$ and $\bar{\Psi}_{\varepsilon}$ analogously to $(2.9)$.

REMARK 2.4. Assume that $\varrho>0$, and consider the function

$$
\mathcal{V}(x):=\left(\varepsilon \theta \bar{\Psi}(-x)+\bar{\Psi}_{\varepsilon}(x)\right)^{p}
$$

for some $p \geq 1$. Then it follows directly from the proofs of Lemma 2.1 and Theorem 2.1 that there exist positive constants $\varepsilon, \theta, \bar{\kappa}_{0}, \bar{\kappa}_{1}$, and a cube $K \subset \mathbb{R}^{m}$, depending only on $p$, such that

$$
\mathcal{L}_{u} \mathcal{V}(x) \leq \begin{cases}\bar{\kappa}_{0} \mathbf{1}_{K}(x)-\bar{\kappa}_{1} \mathcal{V}(x) & \forall x \in \mathcal{K}_{0}^{-} \\ -p \varepsilon \frac{\varrho}{2 m}(\mathcal{V}(x))^{\frac{p-1}{p}} & \forall(x, u) \in \mathcal{K}_{0}^{+} \times \Delta\end{cases}
$$

In Theorem 2.2 which follows we do not assume that $\varrho>0$.

Theorem 2.2. Assume that $\Gamma>0$. With $\bar{C}$ as defined in (2.31), let

$$
\theta=\frac{\left(1-\beta_{\min }\right) \vee \frac{1}{2}}{\beta_{\max }}, \quad \text { and } \varepsilon_{0}:=\frac{1}{2 \sqrt{\bar{C}}}\left[\theta \wedge \beta_{\min }\left(\beta_{\min } \wedge \frac{1}{2}\right)\right] \frac{(1 \wedge \theta) \mu_{\min }}{(1 \vee \theta)^{2} \mu_{\max }}
$$

Then, for any $\varepsilon \leq \varepsilon_{0}$, the function

$$
\widetilde{V}(x):=\exp \left(\frac{1}{2}\left[\Psi_{\epsilon, \theta}^{*}(x)\right]^{2}\right)=\exp \left(\frac{1}{2}\left[\varepsilon \theta \Psi(-x)+\Psi_{\varepsilon}(x)\right]^{2}\right)
$$

satisfies the Foster-Lyapunov equation

$$
\mathcal{L}_{u} \widetilde{V}(x) \leq \tilde{\kappa}_{0}-\varepsilon^{2}\left[\theta \wedge \beta_{\min }\left(\beta_{\min } \wedge \frac{1}{2}\right)\right] \frac{1 \wedge \theta}{2 \mu_{\max }}\|x\|_{1}^{2} \widetilde{V}(x) \quad \forall(x, u) \in \mathbb{R}^{m} \times \Delta,
$$

for a positive constant $\tilde{\kappa}_{0}$ which depends only on $\varepsilon$ and the system parameters. In particular, the process $X_{t}$ governed by (2.1) is uniformly exponentially ergodic, and the associated invariant probability measures have sub-Gaussian tails.

Proof. We borrow some calculations from the proof of Lemma 2.1. Using (2.24), and scaling this with the new definition of $\theta$ in (2.38), we have

$$
\begin{aligned}
\left(\left(1-\beta_{\min }\right) \vee \frac{1}{2}\right)\left(-\sum_{i \in \mathcal{I}} \psi_{\varepsilon}^{\prime}\left(x_{i}\right) x_{i}+\langle e, x\rangle \sum_{i \in \mathcal{I}} \psi_{\varepsilon}^{\prime}\left(x_{i}\right)\left(1-\beta_{i}\right)^{+} u_{i}\right) & \\
& +\varepsilon \theta\langle e, x\rangle \sum_{i \in \mathcal{I}} \psi^{\prime}\left(-x_{i}\right)\left(\beta_{i}-1\right)^{+} u_{i} \leq 0 .
\end{aligned}
$$

Here $\varrho$ is not necessarily positive, so by $(2.11)$ we have

$$
-\frac{\varrho}{m} \sum_{i \in \mathcal{I}} \psi_{\varepsilon}^{\prime}\left(x_{i}\right)+\varepsilon \theta \frac{\varrho}{m} \sum_{i \in \mathcal{I}} \psi^{\prime}\left(-x_{i}\right)+\varepsilon \theta \sum_{i \in \mathcal{I}} \psi^{\prime}\left(-x_{i}\right) x_{i} \leq \varepsilon\left(|\varrho|+\theta|\varrho|+\theta \frac{m}{2}-\theta\left\|x^{-}\right\|_{1}\right)
$$

on $\mathbb{R}^{m}$. Note that

$$
\langle e, x\rangle \sum_{i \in \mathcal{I}} \psi_{\varepsilon}^{\prime}\left(x_{i}\right)\left(1-\beta_{i}\right)^{+} u_{i} \leq\left\|x^{+}\right\|_{1}\left(1-\beta_{\min }\right) \sum_{i \in \mathcal{I}} \psi_{\varepsilon}^{\prime}\left(x_{i}\right) u_{i} \leq\left\|x^{+}\right\|_{1}\left(1-\beta_{\min }\right) .
$$


Thus, using (2.11), we have

$$
\left(\beta_{\min } \wedge \frac{1}{2}\right)\left(-\sum_{i \in \mathcal{I}} \psi_{\varepsilon}^{\prime}\left(x_{i}\right) x_{i}+\langle e, x\rangle \sum_{i \in \mathcal{I}} \psi_{\varepsilon}^{\prime}\left(x_{i}\right)\left(1-\beta_{i}\right)^{+} u_{i}\right) \leq \varepsilon\left(\beta_{\min } \wedge \frac{1}{2}\right)\left(\frac{m}{2 \varepsilon}-\beta_{\text {min }}\left\|x^{+}\right\|_{1}\right) .
$$

Let $\bar{\theta}:=\theta \wedge \beta_{\min }\left(\beta_{\min } \wedge \frac{1}{2}\right)$. Adding (2.40)-(2.42), using (2.13a) and (2.13b), and also (2.10), we obtain

$$
\begin{aligned}
\left.\Psi_{\varepsilon, \theta}^{*}(x)\left\langle\nabla \Psi_{\varepsilon, \theta}^{*}(x), b(x, u)\right\rangle \leq \varepsilon\left(|\varrho|+\theta|\varrho|+\theta \frac{m}{2}+\left(\beta_{\min } \wedge \frac{1}{2}\right) \frac{m}{2 \varepsilon}-\bar{\theta}\|x\|_{1}\right)\right) \Psi_{\varepsilon, \theta}^{*}(x) \\
\leq \varepsilon\left(|\varrho|+\theta|\varrho|+\theta \frac{m}{2}+\left(\beta_{\min } \wedge \frac{1}{2}\right) \frac{m}{2 \varepsilon}\right) \frac{1 \vee \theta}{\mu_{\min }}\|x\|_{1} \\
-\varepsilon^{2} \bar{\theta}\left(\frac{1 \wedge \theta}{\mu_{\max }}\|x\|_{1}-\frac{m}{2 \varepsilon}\right)\|x\|_{1} \\
\leq \varepsilon \hat{c}_{0}-\varepsilon^{2} \bar{\theta} \frac{1 \wedge \theta}{\mu_{\max }}\|x\|_{1}^{2} \quad \forall(x, u) \in \mathcal{K}_{0}^{+} \times \Delta,
\end{aligned}
$$

where

$$
\hat{c}_{0}:=\left(|\varrho|+\theta|\varrho|+\theta \frac{m}{2}+\left(\beta_{\min } \wedge \frac{1}{2}\right) \frac{m}{2 \varepsilon}\right) \frac{1 \vee \theta}{\mu_{\min }}+\frac{m}{2} \bar{\theta} .
$$

It is straightforward to verify that $(2.43)$ is also valid on $\mathcal{K}_{0}^{-} \times \Delta$. Following the proof of Theorem 2.1, we have

$$
\begin{aligned}
\operatorname{trace}\left(a \nabla^{2} \widetilde{V}(x)\right) & \leq\left[\frac{3}{2} \varepsilon(\varepsilon+\theta) \Psi_{\varepsilon, \theta}^{*}(x)+\varepsilon^{2}(1 \vee \theta)^{2} \bar{C}\left(1+\left(\Psi_{\varepsilon, \theta}^{*}(x)\right)^{2}\right)\right] \widetilde{V}(x) \\
& \leq \varepsilon\left[\varepsilon(1 \vee \theta)^{2} \bar{C}+\frac{3}{2} \varepsilon(\varepsilon+\theta) \frac{1 \vee \theta}{\mu_{\min }}\|x\|_{1}+\varepsilon^{3} \bar{C} \frac{(1 \vee \theta)^{4}}{\mu_{\min }^{2}}\|x\|_{1}^{2}\right] \widetilde{V}(x) .
\end{aligned}
$$

Combining (2.43) and (2.44), we obtain

$$
\begin{array}{r}
\operatorname{trace}\left(a \nabla^{2} \widetilde{V}(x)\right)+\langle\nabla \widetilde{V}(x), b(x, u)\rangle \leq\left[\varepsilon^{2}(1 \vee \theta)^{2} \bar{C}+\varepsilon\left(\frac{3}{2}(\varepsilon+\theta) \frac{1 \vee \theta}{\mu_{\min }}+\hat{c}_{0}\right)\|x\|_{1}\right. \\
\left.-\varepsilon^{2}\left(\bar{\theta} \frac{1 \wedge \theta}{\mu_{\max }}-\varepsilon^{2} \bar{C} \frac{(1 \vee \theta)^{4}}{\mu_{\min }^{2}}\right)\|x\|_{1}^{2}\right] \widetilde{V}(x),
\end{array}
$$

from which the validity of $(2.39)$ on $\mathcal{K}_{0}^{+} \times \Delta$ follows by selecting $\varepsilon$ sufficiently small. Verifying the validity of $(2.39)$ on $\mathcal{K}_{0}^{-} \times \Delta$, is simpler, and is a straightforward application of $(2.10),(2.11)$, and (2.44). This finishes the proof.

REMARK 2.5. The counterpart of Remark 2.4 applies relative to Theorem 2.2. In particular, the function $\mathcal{V}$ in (2.37) for $p>0$ is a Lyapunov function. Indeed, there exist positive constants $\varepsilon$, $\theta, \check{\kappa}_{0}$ and $\check{\kappa}_{1}$, and a cube $K \subset \mathbb{R}^{m}$, depending only on $p$, such that

$$
\mathcal{L}_{u} \mathcal{V}(x) \leq \check{\kappa}_{0} \mathbf{1}_{K}(x)-\check{\kappa}_{1} \mathcal{V}(x) \quad \forall(x, u) \in \mathcal{K}_{0}^{+} \times \Delta .
$$

REMARK 2.6. It is worth noting that if $\Gamma>0$, then by choosing $\theta>0$ as in (2.38), the function

$$
\breve{V}(x):=\exp (\eta \theta \Psi(-x)+\eta \Psi(x))
$$

satisfies

$$
\mathcal{L}_{u} \breve{V}(x) \leq \breve{\kappa}_{0}-\breve{\kappa}_{1}\|x\|_{1} \breve{V}(x) \quad \forall(x, u) \in \mathcal{K}_{0}^{+} \times \Delta,
$$

for all $\eta>0$, and for some positive constants $\breve{\kappa}_{0}$ and $\breve{\kappa}_{1}$ depending only on $\eta$. Indeed, using (2.13a), $(2.13 \mathrm{~b})$, and $(2.40)$, we deduce, with $\hat{\theta}:=1-\left(\left(1-\beta_{\min }\right) \vee \frac{1}{2}\right)$, that

$$
\begin{aligned}
\frac{1}{\eta \breve{V}(x)}\langle\nabla \breve{V}(x), b(x, u)\rangle & =\frac{\varrho}{m} \sum_{i \in \mathcal{I}}\left(\theta \psi^{\prime}\left(-x_{i}\right)-\psi^{\prime}\left(x_{i}\right)\right)-\sum_{i \in \mathcal{I}}\left((1-\hat{\theta}) \psi_{\varepsilon}^{\prime}\left(x_{i}\right) x_{i}-\theta \psi_{\varepsilon}^{\prime}\left(-x_{i}\right)\right) x_{i} \\
& +(1-\hat{\theta})\langle e, x\rangle^{+} \sum_{i \in \mathcal{I}} \psi^{\prime}\left(x_{i}\right)\left(1-\beta_{\min }\right)^{+} u_{i} \\
& \leq \frac{m}{2}(1-\hat{\theta}+\theta)+\frac{\varrho}{m} \sum_{i \in \mathcal{I}}\left(\theta \psi^{\prime}\left(-x_{i}\right)-\psi^{\prime}\left(x_{i}\right)\right)-\left(\beta_{\min }(1-\hat{\theta}) \wedge \theta\right)\|x\|_{1},
\end{aligned}
$$

where we also used (2.11) and (2.12). The rest is routine. 
2.6. Results concerning the tail of the invariant distribution. Gamarnik and Stolyar in [20] conjecture that, provided $\varrho>0, \exp \left(\theta \sum_{i} x_{i}^{-}\right)$is integrable under an invariant probability measure for all $\theta>0$. They prove this when $\gamma_{i} \leq \mu_{i}$ for all $i \in \mathcal{I}$ [20, Theorem $\left.2(\mathrm{i})\right]$. The proof is for the diffusion-scaled queueing processes, and relies on a simple comparison to a system with infinitely many servers. For this proof to go through though, it seems necessary that all $i$ satisfy $\gamma_{i} \leq \mu_{i}$. We improve upon this result, by showing that $\mathrm{e}^{\theta x_{i}^{-}}$is integrable under an invariant probability measure for all $\theta>0$, for any $i$ such that $\gamma_{i} \leq \mu_{i}$. Of course this proof applies to the limiting diffusion, but we show in Section 3 how to recover this property for the prelimit in Theorem 3.5. The general conjecture remains open.

We need some notation. We let

$$
\mathcal{I}_{1}:=\left\{i \in \mathcal{I}: \gamma_{i} \leq \mu_{i}\right\}
$$

and for a positive constant $\eta$, we define

$$
\Phi_{1}(x):=\sum_{i \in \mathcal{I}_{1}} \frac{\psi\left(-x_{i}\right)}{\mu_{i}}, \quad \text { and } \mathcal{V}_{1}(x):=\exp \left(\eta \Phi_{1}(x)\right)
$$

Lemma 2.2. Assume that $\varrho>0$. Let $\eta>0$ be arbitrary, and $V(x)=\exp \left(\Psi_{\varepsilon_{0}, \theta}^{*}(x)\right)$, with $\varepsilon_{0}$ as in Theorem 2.1, and the constant $\theta$ chosen to satisfy (2.15) and (2.27). Then

$$
\mathcal{L}_{u}\left(\mathcal{V}_{1}+V\right)(x) \leq \begin{cases}\kappa_{0} \mathbf{1}_{K}(x)-\kappa_{1}\|x\|_{1}\left(\mathcal{V}_{1}(x)+V(x)\right) & \forall x \in \mathcal{K}_{0}^{-}, \\ \kappa_{0} \mathbf{1}_{K}(x)-\varepsilon_{0} \frac{\varrho}{8 m}\left(\mathcal{V}_{1}(x)+V(x)\right) & \forall(x, u) \in \mathcal{K}_{0}^{+} \times \Delta\end{cases}
$$

for some positive constants $\kappa_{0}$ and $\kappa_{1}$, and some cube $K \in \mathbb{R}^{m}$.

Proof. Using (2.13a) and (2.13b), we write

$$
\begin{gathered}
\frac{1}{\mathcal{V}_{1}(x)}\left\langle\nabla \mathcal{V}_{1}(x), b(x, u)\right\rangle=\frac{1}{2} \eta\left|\mathcal{I}_{1}\right|+\eta \frac{\varrho}{m} \sum_{i \in \mathcal{I}_{1}} \psi^{\prime}\left(-x_{i}\right)-\eta\left(\frac{1}{2}\left|\mathcal{I}_{1}\right|-\sum_{i \in \mathcal{I}_{1}} \psi^{\prime}\left(-x_{i}\right) x_{i}\right) \\
-\eta\langle e, x\rangle^{+} \sum_{i \in \mathcal{I}_{1}}\left(1-\beta_{i}\right) \psi^{\prime}\left(-x_{i}\right) u_{i} .
\end{gathered}
$$

Let

$$
H(x):=\operatorname{trace}\left(a \nabla^{2} \Phi(x)\right)+\left\langle\nabla \Phi(x), a\left(\nabla \Phi(x)+2 \nabla \Psi_{\varepsilon, \theta}^{*}(x)\right)\right\rangle .
$$

Recall the definition in (2.36). It is clear from (2.8) that we can select $\delta \in(0,1)$ and $r>0$ such that

$$
\begin{aligned}
H(x) \mathcal{V}_{1}(x)+\left(\eta \frac{m}{2}+\eta \varrho\right) \mathcal{V}_{1}(x) & \leq \varepsilon_{0} \frac{\varrho}{4 m} V(x), \\
\text { and } \mathcal{V}_{1}(x) & \leq V(x) \quad \forall x \in K_{r}^{\mathrm{c}} \cap \mathcal{K}_{\delta}^{+}
\end{aligned}
$$

Combining (2.28) and (2.48), we obtain

$$
\mathcal{L}_{u}\left(\mathcal{V}_{1}+V\right)(x) \leq \kappa_{0}-\frac{\varepsilon_{0}}{2}\left(\frac{\varrho}{4 m}+\theta\left\|x^{-}\right\|_{1}\right)\left(\mathcal{V}_{1}(x)+V(x)\right) \quad \forall x \in K_{r}^{\mathrm{c}} \cap \mathcal{K}_{\delta}^{+},
$$

and all $u \in \Delta$. By (2.47), we have

$$
\frac{1}{\mathcal{V}_{1}(x)}\left\langle\nabla \mathcal{V}_{1}(x), b(x, u)\right\rangle \leq \eta\left(\frac{m}{2}+\varrho\right)-\eta \sum_{i \in \mathcal{I}_{1}} x_{i}^{-} .
$$


Consider the set

$$
\mathcal{K}:=\left\{x \in \mathcal{K}_{0}^{+} \backslash \mathcal{K}_{\delta}^{+}: \frac{1}{2} \eta \sum_{i \in \mathcal{I}_{1}} x_{i}^{-} \leq \eta\left(\frac{m}{2}+\varrho\right)+H(x)+\varepsilon_{0} \frac{\varrho}{4 m}\right\} .
$$

Since $H$ is bounded on $\mathbb{R}^{m}$, it is clear by the definition of $\mathcal{K}$ that $\mathcal{V}_{1}$ and $\mathcal{L}_{u} \mathcal{V}_{1}$ are both bounded on $\mathcal{K}$. Therefore, since $V$ is coercive on $\mathcal{K}$, that is, $\liminf _{\{|x| \rightarrow \infty, x \in \mathcal{K}\}} V(x) \rightarrow \infty$, there exists $r_{\circ}>0$ such that

$$
\left|\mathcal{L}_{u} \mathcal{V}_{1}(x)\right| \leq \varepsilon_{0} \frac{\varrho}{4 m} V(x), \quad \text { and } \quad \mathcal{V}_{1}(x) \leq \mathcal{V}(x) \quad \forall(x, u) \in\left(\mathcal{K} \cap K_{r_{\circ}}^{\mathrm{c}}\right) \times \Delta
$$

On the other hand, we have

$$
\mathcal{L}_{u} \mathcal{V}_{1}(x) \leq-\left(\varepsilon_{0} \frac{\varrho}{4 m}+\frac{\eta}{2}\left\|x^{-}\right\|_{1}\right) \mathcal{V}_{1}(x) \quad \forall(x, u) \in\left(\mathcal{K}_{0}^{+} \backslash \mathcal{K}_{\delta}^{+}\right) \cap \mathcal{K}^{c}
$$

by (2.50). Equations (2.51) and (2.52), together with (2.28) and (2.49), imply that

$$
\mathcal{L}_{u}\left(\mathcal{V}_{1}+V\right)(x) \leq \kappa_{0}-\frac{\varepsilon_{0}}{2}\left(\frac{\varrho}{4 m}+\left(\theta \wedge \frac{\eta}{2}\right)\left\|x^{-}\right\|_{1}\right)\left(\mathcal{V}_{1}(x)+V(x)\right) \quad \forall x \in K_{r \vee r_{\circ}}^{\mathrm{c}} \cap \mathcal{K}_{0}^{+} .
$$

The estimate on $\mathcal{K}_{0}^{-}$is straightforward. Indeed, (2.47) shows that $\mathcal{V}_{1}$ satisfies this estimate, and (2.28) asserts the same for $V$. This completes the proof.

The following is immediate from Lemma 2.2.

Corollary 2.1. Suppose $\varrho>0$. Then the function $\exp \left(\eta \sum_{i \in \mathcal{I}_{1}} \frac{\psi\left(-x_{i}\right)}{\mu_{i}}\right)$ is integrable under the invariant distribution for any $\eta>0$.

In $[20$, Theorem 4(i)] it is shown that if $\nu$ is any limit of the invariant distributions of the diffusion-scaled queueing processes, then there exists some $\theta$ such that $f(x)=\exp \left(\theta \sum_{i}\left(x_{i}^{-}\right)^{2}\right)$ is integrable under $\nu$. As is pointed out in [20], this property holds only at the limit. The function $f$ is not integrable under the stationary distribution of the prelimit model. The proof is rather tedious and is approached via truncations (see [20, Proposition 12]). In what follows, we provide a simple proof of this result, by showing that this property holds for the limiting diffusion.

Recall the definitions in (2.45) and (2.46).

Theorem 2.3. Assume that $\varrho>0$, and let

$$
\Phi_{\eta}(x):=\sum_{i \in \mathcal{I}_{1}} \frac{\psi_{\eta}\left(-x_{i}\right)}{\mu_{i}}, \quad \widetilde{\mathcal{V}}_{\eta}(x):=\exp \left(\frac{1}{2}\left[\eta \Phi_{\eta}(x)\right]^{2}\right), \quad \text { and } \quad V(x):=\exp \left(\Psi_{\varepsilon_{0}, \theta}^{*}(x)\right),
$$

with $\varepsilon_{0}$ and $\theta$ chosen as in Lemma 2.2. Then there exists $\eta>0$, such that the function $\mathcal{V}:=\widetilde{\mathcal{V}}_{\eta} V$ satisfies

$$
\mathcal{L}_{u} \mathcal{V}(x) \leq c_{0}-c_{1} \mathcal{V}(x) \quad \forall(x, u) \in \mathbb{R}^{m} \times \mathbb{U} .
$$

Proof. As in (2.47), we have

$$
\begin{aligned}
\left\langle\nabla \Phi_{\eta}(x), b(x, u)\right\rangle=\frac{1}{2}\left|\mathcal{I}_{1}\right| & +\frac{\varrho}{m} \sum_{i \in \mathcal{I}_{1}} \psi_{\eta}^{\prime}\left(-x_{i}\right)-\left(\frac{1}{2}\left|\mathcal{I}_{1}\right|-\sum_{i \in \mathcal{I}_{1}} \psi_{\eta}^{\prime}\left(-x_{i}\right) x_{i}\right) \\
& -\langle e, x\rangle^{+} \sum_{i \in \mathcal{I}_{1}}\left(1-\beta_{i}\right) \psi_{\eta}^{\prime}\left(-x_{i}\right) u_{i} \quad \forall(x, u) \in \mathbb{R}^{m} \times \Delta .
\end{aligned}
$$

Let

$$
\widetilde{H}_{\eta}(x):=\frac{1}{2} \operatorname{trace}\left(a \nabla^{2}\left[\Phi_{\eta}(x)\right]^{2}\right)+\frac{1}{2}\left\langle\nabla\left[\Phi_{\eta}(x)\right]^{2}, a\left(\nabla\left[\Phi_{\eta}(x)\right]^{2}+2 \nabla \Psi_{\varepsilon, \theta}^{*}(x)\right)\right\rangle .
$$


Note that $\widetilde{H}_{\eta}(x) \leq c_{0} \eta^{2}+c_{1} \eta^{4}\left[\Phi_{\eta}(x)\right]^{2}$ for some positive constants $c_{0}+c_{1}$. Consider the set

$$
\widetilde{\mathcal{K}}:=\left\{x \in \mathcal{K}_{0}^{+}: \eta \sum_{i \in \mathcal{I}_{1}} x_{i}^{-} \leq \frac{\left|\mathcal{I}_{1}\right|}{2}+\eta \varrho+\left(\eta^{2} \widetilde{H}_{\eta}(x)+\varepsilon_{0} \eta \frac{\varrho}{4 m}\right)\left[\Phi_{\eta}(x)\right]^{-1}\right\} .
$$

It is clear that $\Phi_{\eta}(x)$ is bounded on this set, and thus the same applies to $\widetilde{H}_{\eta}$, and $\left\langle\nabla \Phi_{\eta}(x), b(x, u)\right\rangle$. Thus we have

$$
\sup _{x \in \widetilde{\mathcal{K}}}\left[\eta^{2} \widetilde{H}_{\eta}(x)+\eta^{2} \Phi_{\eta}(x)\left\langle\nabla \Phi_{\eta}(x), b(x, u)\right\rangle\right] \underset{\eta \searrow 0}{\longrightarrow} 0 .
$$

However, (2.28) and (2.54) imply that $\eta$ may be selected small enough so that

$$
\mathcal{L}_{u} \mathcal{V}(x) \leq \kappa_{0}-\varepsilon\left(\frac{\varrho}{4 m}+\theta\left\|x^{-}\right\|_{1}\right) \mathcal{V}(x) \quad \forall(x, u) \in\left(\mathcal{K}_{0}^{+} \cap \widetilde{\mathcal{K}}\right) \times \Delta .
$$

On the other hand, by (2.53) and the definition of $\widetilde{\mathcal{K}}$, we have

$$
\eta^{2} \widetilde{H}_{\eta}(x)+\eta^{2} \Phi_{\eta}(x)\left\langle\nabla \Phi_{\eta}(x), b(x, u)\right\rangle \leq 0 \quad \forall(x, u) \in\left(\mathcal{K}_{0}^{+} \cap \widetilde{\mathcal{K}}^{c}\right) \times \Delta
$$

which also implies $(2.55)$ on $\left(\mathcal{K}_{0}^{+} \cap \widetilde{\mathcal{K}}^{c}\right) \times \Delta$. Since the bound on $\mathcal{K}^{+}$is clear, this completes the proof.

3. Uniform ergodicity of multiclass many-server queues. For a detailed description of this model, see [3]. Here we only review the basic structure which is used for our results. We consider a sequence of $G I / M / n+M$ queues with $m$ classes of customers, indexed by $n$, which is the number of servers. Customers of each class form their own queue and are served in the order of their arrival.

3.1. Model and assumptions. Let $A_{i}^{n}, i \in \mathcal{I}=\{1, \ldots, m\}$, denote the arrival process of class- $i$ customers with arrival rate $\lambda_{i}^{n}$. We assume that $\left\{A_{i}^{n}\right\}_{i \in \mathcal{I}}$ are renewal processes defined as follows. Let $\left\{R_{i j}: i \in \mathcal{I}, j \in \mathbb{N}\right\}$ be a collection of independent positive random variables such that, for each $i \in \mathcal{I},\left\{R_{i j}\right\}_{j \in \mathbb{N}}$ have a common distribution function $F_{i}$ having a density $f_{i}$, mean equal to 1 , and squared coefficient of variation $(\mathrm{SCV}) c_{a, i}^{2} \in(0, \infty)$. Let

$$
h_{i}(\tau):=\frac{f_{i}(\tau)}{1-F_{i}(\tau)}, \quad \text { and } \quad \zeta_{i}(\tau):=\frac{\int_{\tau}^{\infty}\left(1-F_{i}(r)\right) \mathrm{d} r}{1-F_{i}(\tau)}
$$

for $\tau \geq 0$, denote the hazard rate and the mean residual life functions for each $i \in \mathcal{I}$, respectively. The arrival process $A_{i}^{n}$ is then given by

$$
A_{i}^{n}(t):=\max \left\{k \geq 0: \sum_{j=1}^{k} R_{i j} \leq \lambda_{i}^{n} t\right\}, \quad t \geq 0, i \in \mathcal{I} .
$$

We assume the following structural hypotheses on the collection $\left\{F_{i}\right\}_{i \in \mathcal{I}}$, which are enforced in this subsection without further mention.

Assumption 3.1. The distribution functions $\left\{F_{i}\right\}_{i \in \mathcal{I}}$ satisfy $F_{i}(0)=0$, and have a locally bounded density $f_{i}$ with unbounded support. In addition, the mean residual life functions $\left\{\zeta_{i}\right\}_{i \in \mathcal{I}}$ are bounded. 
The service and patience times are exponentially distributed, with class-dependent rates, $\mu_{i}$ and $\gamma_{i}$, respectively, for class- $i$ customers. The arrival, service and abandonment processes of each class are mutually independent.

The queueing process (counting the number both in service and in queue for each class) of the $n^{\text {th }}$ system $X^{n}=\left\{X^{n}(t): t \geq 0\right\}$ is governed by

$$
X_{i}^{n}(t)=X_{i}^{n}(0)+A_{i}^{n}(t)-Y_{i}^{n}\left(\mu_{i}^{n} \int_{0}^{t} Z_{i}^{n}(s) \mathrm{d} s\right)-R_{i}^{n}\left(\gamma_{i}^{n} \int_{0}^{t} Q_{i}^{n}(s) \mathrm{d} s\right)
$$

for $i \in \mathcal{I}$ and $t \geq 0$. Here $Y_{i}^{n}$ and $R_{i}^{n}$, are mutually independent rate-1 Poisson processes, independent of the initial conditions $X_{i}^{n}(0)$ and the arrival processes $A_{i}^{n}$, for all $i \in \mathcal{I}$. Also, $Z_{i}^{n}(s)$ and $Q_{i}^{n}(s)$ represent the numbers of class- $i$ jobs in service and in queue at time $s, s \geq 0$, respectively.

3.1.1. The Halfin-Whitt regime. The parameters satisfy the following limits as $n \rightarrow \infty$ for all $i \in \mathcal{I}$ :

$$
\begin{gathered}
\frac{\lambda_{i}^{n}}{n} \rightarrow \lambda_{i}>0, \quad \mu_{i}^{n} \rightarrow \mu_{i}>0, \quad \gamma_{i}^{n} \rightarrow \gamma_{i} \geq 0, \\
\frac{\lambda_{i}^{n}-n \lambda_{i}}{\sqrt{n}} \rightarrow \hat{\lambda}_{i}, \quad \sqrt{n}\left(\mu_{i}^{n}-\mu_{i}\right) \rightarrow \hat{\mu}_{i}, \\
\rho_{i}^{n}:=\frac{\lambda_{i}^{n}}{n \mu_{i}^{n}} \rightarrow \rho_{i}:=\frac{\lambda_{i}}{\mu_{i}}<1, \quad \sum_{i=1}^{m} \rho_{i}=1 .
\end{gathered}
$$

The assumptions in (3.1) imply that

$$
\varrho^{n}:=\sqrt{n}\left(1-\sum_{i=1}^{m} \frac{\lambda_{i}^{n}}{n \mu_{i}^{n}}\right) \rightarrow \varrho:=\sum_{i=1}^{m} \frac{\rho_{i} \hat{\mu}_{i}-\hat{\lambda}_{i}}{\mu_{i}} \in \mathbb{R} .
$$

We define the diffusion-scaled variables by

$$
\begin{gathered}
\hat{X}_{i}^{n}(t)=\frac{1}{\sqrt{n}}\left(X_{i}^{n}(t)-\frac{\lambda_{i}^{n}}{\mu_{i}^{n}}\right)-\frac{\varrho^{n}}{m}, \quad \hat{Z}_{i}^{n}(t)=\frac{1}{\sqrt{n}}\left(Z_{i}^{n}(t)-\frac{\lambda_{i}^{n}}{\mu_{i}^{n}}\right)-\frac{\varrho^{n}}{m}, \\
\hat{Q}_{i}^{n}(t)=\frac{1}{\sqrt{n}} Q_{i}^{n}(t), \quad \text { and } \quad \hat{A}_{i}^{n}(t)=\frac{1}{\sqrt{n}}\left(A_{i}^{n}(t)-\lambda_{i}^{n} t\right), \quad i \in \mathcal{I} .
\end{gathered}
$$

Then, we obtain the following representation of $\hat{X}_{i}^{n}(t)$ :

$$
\begin{aligned}
\hat{X}_{i}^{n}(t)=\hat{X}_{i}^{n}(0)-\frac{\varrho^{n} \mu_{i}^{n}}{m} t-\mu_{i}^{n} \int_{0}^{t} \hat{Z}_{i}^{n}(s) \mathrm{d} s- & \gamma_{i}^{n} \int_{0}^{t} \hat{Q}_{i}^{n}(s) \mathrm{d} s \\
& +\hat{A}_{i}^{n}(t)-\hat{M}_{S, i}^{n}(t)-\hat{M}_{R, i}^{n}(t), \quad t \geq 0,
\end{aligned}
$$

where

$$
\begin{aligned}
& \hat{M}_{Y, i}^{n}(t):=\frac{1}{\sqrt{n}}\left(Y_{i}^{n}\left(\mu_{i}^{n} \int_{0}^{t} Z_{i}^{n}(s) \mathrm{d} s\right)-\mu_{i}^{n} \int_{0}^{t} \hat{Z}_{i}^{n}(s) \mathrm{d} s\right), \\
& \hat{M}_{R, i}^{n}(t):=\frac{1}{\sqrt{n}}\left(R_{i}^{n}\left(\gamma_{i}^{n} \int_{0}^{t} Q_{i}^{n}(s) \mathrm{d} s\right)-\gamma_{i}^{n} \int_{0}^{t} Q_{i}^{n}(s) \mathrm{d} s\right),
\end{aligned}
$$

and the last two terms $\hat{M}_{Y, i}^{n}(t)$ and $\hat{M}_{R, i}^{n}(t)$ are square integrable martingales associated with the service and abandonment processes, respectively. The martingales are compensated rate- 1 Poisson processes with random time changes, with respect to the natural filtration, see [3]. 
Note that the diffusion-scaled arrival processes satisfy

$$
\hat{A}^{n} \Rightarrow \operatorname{diag}\left(\lambda_{1} c_{a, 1}^{2}, \ldots, \lambda_{m} c_{a, m}^{2}\right)^{1 / 2} W \quad \text { in }\left(\mathbb{D}_{m}, J_{1}\right) \quad \text { as } n \rightarrow \infty,
$$

where $W$ is a standard $m$-dimensional Wiener process and $\left(\mathbb{D}_{m}, J_{1}\right)$ represents the space of càdlàg functions in $\mathbb{R}^{m}$ endowed with the Skorokhod $J_{1}$ topology. Assuming that $\hat{X}^{n}(0) \Rightarrow X(0)=x_{0}$ for a constant $x_{0} \in \mathbb{R}^{m}$, it then follows that $\hat{X}^{n} \Rightarrow X$ in $\left(\mathbb{D}_{m}, J_{1}\right)$ as $n \rightarrow \infty$, where the limit process $X$ satisfies $(2.1)$ with $\sigma\left(X_{t}\right)=\operatorname{diag}\left(\lambda_{1}\left(1+c_{a, 1}^{2}\right), \ldots, \lambda_{m}\left(1+c_{a, m}^{2}\right)\right)^{1 / 2}$. In the case of Poisson arrivals, we have $c_{a, i}^{2}=1$ and thus $\sigma\left(X_{t}\right)=\operatorname{diag}\left(2 \lambda_{1}, \ldots, 2 \lambda_{m}\right)^{1 / 2}$.

REMARK 3.1. Note that this scaling is different from that used in $[10,26,3]$, where the centering term uses $n \rho_{i}$ for the processes $X_{i}^{n}(t)$ and $Z_{i}^{n}(t)$. Here we use the prelimit parameters $\lambda_{i}^{n} / \mu_{i}^{n}$ together with the "adjustment" $\varrho^{n} / m$, which can be regarded as the "reallocation" of the "safety staffing". Recall that when $\varrho^{n}>0$ (and $\varrho>0$ ), the condition in (3.2) is equivalent to the positive square-root safety staffing rule (see [36]). In addition, the diffusion-scaled process $\hat{X}^{n}$ converges to the limiting diffusion $X$ with the drift given in (2.6). That follows from the standard martingale convergence technique in [31] using the representation of $\hat{X}^{n}$ in (3.4).

3.1.2. Scheduling policies. We define the space

$$
\mathcal{Z}^{n}(x):=\left\{z \in \mathbb{Z}_{+}^{m}: z_{i} \leq x_{i},\|z\|_{1}=n \wedge\|x\|_{1}\right\} .
$$

A scheduling policy is called (stationary) Markov if $Z^{n}(t)=z\left(X^{n}(t), S^{n}(t)\right)$ for some function $z: \mathbb{Z}_{+}^{m} \times \mathbb{R}_{+}^{m} \rightarrow \mathcal{Z}^{n}(x)$, in which case we identify the policy with the function $z$. Let $S^{n}(t)=$ $\left(S_{1}^{n}(t), \ldots, S_{m}^{n}(t)\right)$, where $S_{i}^{n}(t)$ denotes the age process for class- $i$ customers, defined by

$$
S_{i}^{n}(t):=t-\frac{1}{\lambda_{i}^{n}} \sum_{j=1}^{A_{i}^{n}(t)} R_{i j}, \quad t \geq 0 .
$$

Let

$$
r_{i}^{n}\left(s_{i}\right):=\lambda_{i}^{n} \frac{f_{i}\left(\lambda_{i}^{n} s_{i}\right)}{1-F_{i}\left(\lambda_{i}^{n} s_{i}\right)}, \quad s_{i} \geq 0,
$$

denote the scaled hazard rate function for the interarrival times of $A_{i}^{n}(t)$.

Under a Markov policy, the process $\left(X^{n}, S^{n}\right)$ is Markov with extended generator

$$
\begin{aligned}
\mathcal{A}_{z}^{n} g(x, s):=\sum_{i \in \mathcal{I}} \frac{\partial g(x, s)}{\partial s_{i}}+\sum_{i \in \mathcal{I}} r_{i}^{n}( & \left.s_{i}\right)\left(g\left(x+e_{i}, s-s_{i} e_{i}\right)-g(x, s)\right) \\
& +\sum_{i \in \mathcal{I}}\left(\mu_{i}^{n} z_{i}+\gamma_{i}^{n} q_{i}(x, z)\right)\left(g\left(x-e_{i}, s\right)-g(x, s)\right),
\end{aligned}
$$

for $g \in \mathcal{C}_{b}\left(\mathbb{R}^{m} \times \mathbb{R}^{m}\right)$ and $(x, s) \in \mathbb{Z}_{+}^{m} \times \mathbb{R}_{+}^{m}$. Here, $q_{i}(x, z)=x_{i}-z_{i}$, and $e_{i} \in \mathbb{R}^{m}$ denotes the vector with the $i^{\text {th }}$ element equal to 1 and the rest of its elements equal to 0.

Let

$$
\hat{x}_{i}^{n}(x):=\frac{1}{\sqrt{n}}\left(x_{i}-\frac{\lambda_{i}^{n}}{\mu_{i}^{n}}\right)-\frac{\varrho^{n}}{m}, \quad \hat{z}_{i}^{n}(x):=\frac{1}{\sqrt{n}}\left(z_{i}-\frac{\lambda_{i}^{n}}{\mu_{i}^{n}}\right)-\frac{\varrho^{n}}{m}, \text { and } \quad \hat{q}_{i}^{n}(x, z):=\frac{q_{i}(x, z)}{\sqrt{n}} .
$$

We let $X^{n}$ denote the state space of the process $\hat{X}^{n}$. This is a countable subset of $\mathbb{R}^{m}$. Since $x \mapsto \hat{x}^{n}(x)$ is invertible, the set $\mathcal{Z}^{n}(x)$ can be equivalently written as a function of $\hat{x}^{n}$, and abusing the notation we write this as $\mathcal{Z}^{n}\left(\hat{x}^{n}\right)$. In order to keep the notation simple, we often drop the superscript $n$ from $\hat{x}^{n}$, when this is used to denote a generic element of $X^{n}$. 
3.2. Results with renewal arrivals. The first main result is Theorem 3.1 below which is the counterpart of Theorem 2.1 for the $n^{\text {th }}$ system. In order to state this theorem and demonstrate its proof, we need some additional notation which we introduce next.

Let $V$ be the function in (2.16) with $\mu^{n}$ replacing $\mu$ in its definition, and parameters $\varepsilon>0$ and $\theta \in(0,1)$. Let

$$
\zeta_{i}^{n}(\tau):=\zeta_{i}\left(\lambda_{i}^{n} \tau\right), \quad \tau \geq 0, i \in \mathcal{I}
$$

In Theorem 3.1 below, we use the Lyapunov function $\mathcal{V}^{n}$ defined by

$$
\mathcal{V}^{n}(\hat{x}, s):=G^{n}(\hat{x}, s)+V(\hat{x}), \quad(\hat{x}, s) \in X^{n} \times \mathbb{R}_{+}^{m},
$$

with

$$
G^{n}(\hat{x}, s):=\sum_{i \in \mathcal{I}}\left(1-\zeta_{i}^{n}\left(s_{i}\right)\right)\left(V\left(\hat{x}+n^{-1 / 2} e_{i}\right)-V(\hat{x})\right), \quad(\hat{x}, s) \in X^{n} \times \mathbb{R}_{+}^{m} .
$$

Note that, by Assumption 3.1, for any fixed $\theta$, we can choose $\tilde{\varepsilon}_{0}=\tilde{\varepsilon}_{0}(\theta)>0$ small enough so that

$$
\varepsilon\left|\sum_{i \in \mathcal{I}} \frac{1}{\mu_{i}^{n}}\left(1-\zeta_{i}^{n}\left(s_{i}\right)\right)\left(\theta \psi^{\prime}\left(-y_{i}\right)+\psi^{\prime}\left(y_{i}\right)\right)\right| \leq \frac{1}{2} \quad \forall \varepsilon \leq \tilde{\varepsilon}_{0}(\theta), \forall(y, s) \in \mathbb{R}^{m} \times \mathbb{R}_{+}^{m}, \forall n \in \mathbb{N} .
$$

Then, provided $\varepsilon \leq \tilde{\varepsilon}_{0}(\theta)$, we have

$$
\frac{1}{2} V(y) \leq \mathcal{V}^{n}(y, s) \leq \frac{3}{2} V(y) .
$$

We define

$$
\widehat{V}^{n}(x):=V\left(\hat{x}^{n}(x)\right), \quad \text { and } \widehat{G}^{n}(x, s):=\sum_{i \in \mathcal{I}}\left(1-\zeta_{i}^{n}\left(s_{i}\right)\right)\left(V\left(\hat{x}^{n}\left(x+e_{i}\right)\right)-V\left(\hat{x}^{n}(x)\right)\right)
$$

for $x \in \mathbb{Z}_{+}^{m}$. Then the generator $\widehat{\mathcal{A}}_{z}^{n}$ of the diffusion-scaled state process $\left(\hat{X}^{n}, S^{n}\right)$ under a policy $z$ takes the form

$$
\widehat{\mathcal{A}}_{z}^{n} V(\hat{x}, s)=\mathcal{A}_{z}^{n} \widehat{V}^{n}(x, s), \quad \text { and } \widehat{\mathcal{A}}_{z}^{n} G(\hat{x}, s)=\mathcal{A}_{z}^{n} \widehat{G}^{n}(x, s),
$$

where $\mathcal{A}_{z}^{n}$ is as defined in (3.6).

We need to introduce some constants used in the results. First, for a function $f$ on $\mathbb{R}^{m}$, if we define

$$
\mathfrak{d} f(x ; y):=f(x+y)-f(x),
$$

it then follows by a repeated use of the mean value theorem that there exists a constant $\widehat{C}_{1}$ such that

$$
\left|\mathfrak{d} \widehat{V}^{n}\left(x \pm e_{j} ; \pm e_{i}\right)-\mathfrak{d} \widehat{V}^{n}\left(x ; \pm e_{i}\right)\right| \leq \frac{1}{n} \widehat{C}_{1} \varepsilon(\varepsilon+\theta) \widehat{V}^{n}(x) \quad \forall i, j \in \mathcal{I},
$$

and the same bound holds for $\left|\mathfrak{d} \widehat{V}^{n}\left(x ; e_{i}\right)+\mathfrak{d} \widehat{V}^{n}\left(x ;-e_{i}\right)\right|$. Also, by Assumption 3.1, (3.5), and the convergence of the parameters in (3.1), there exists a constant $\widehat{C}_{0}^{n}$ depending on $n$ (implicitly through $\lambda_{i}^{n}$ ), such that

$$
\sup _{n \in \mathbb{N}} \max _{i \in \mathcal{I}}\left(\frac{r_{i}^{n}(\tau)}{n} \vee\left(1+\zeta_{i}^{n}(\tau)\right)\right) \leq \widehat{C}_{0}^{n} \quad \forall \tau \geq 0
$$

We define

$$
\widetilde{C}_{0}^{n}:=m^{2} \widehat{C}_{0}^{n} \widehat{C}_{1}, \quad \widetilde{C}_{1}^{n}:=\widehat{C}_{1}\left(m^{2} \widehat{C}_{0}^{n} \mu_{i}^{n}+m(m-1)\left(\widehat{C}_{0}^{n}\right)^{2}+\sum_{i \in \mathcal{I}} \frac{\lambda_{i}^{n}}{n}\right),
$$


and

$$
\theta_{0}(n):=\frac{1}{1+\left(\beta_{\max }^{n}-1\right)^{+}} \wedge \frac{1}{2 \mu_{\max }^{n}\left(\widetilde{C}_{0}^{n}+\widehat{C}_{1}\right)} \wedge \frac{\varrho^{n}}{m}\left(m+2 \varrho^{n}+4\left(\widetilde{C}_{1}^{n}+m \widehat{C}_{1} \widehat{C}_{2}^{n}+m \widehat{C}_{3}^{n}\right)\right)^{-1} .
$$

Recall $\tilde{\varepsilon}_{0}(\theta)$ in $(3.9)$. We are ready to state the first main result of this section.

Theorem 3.1. We enforce Assumption 3.1, and, in addition, we assume that the hazard rate functions $\left\{h_{i}\right\}_{i \in \mathcal{I}}$ are bounded. Suppose $\varrho^{n}>0$. Then there exists a positive constant $C_{0}^{n}(\varepsilon)$, such that the function $\mathcal{V}^{n}$ in (3.8), with parameters $\theta=\theta_{0}(n)$ and any $\varepsilon<\theta_{0}(n) \wedge \tilde{\varepsilon}_{0}(\theta)$, satisfies

$$
\widehat{\mathcal{A}}_{z}^{n} \mathcal{V}^{n}(\hat{x}, s) \leq C_{0}^{n}(\varepsilon)-\varepsilon \frac{\varrho^{n}}{3 m} \mathcal{V}^{n}(\hat{x}, s) \quad \forall(\hat{x}, s) \in X^{n} \times \mathbb{R}_{+}^{m}, \forall z \in \mathcal{Z}^{n}(\hat{x})
$$

In particular, under any work-conserving stationary Markov policy, the process $\left(\hat{X}^{n}, S^{n}\right)$ is positive Harris recurrent, and $V(\hat{x})$ is integrable under its invariant probability distribution.

REMARK 3.2. It is clear from the Foster-Lyapunov equation (3.17), that the stability result in Theorem 3.1 holds for all $n \in \mathbb{N}$ such that $\varrho^{n}>0$, and the same applies to Theorem 3.3 and Corollary 3.1. We want to emphasize that this is an important byproduct of the approach in this paper. One should compare it to [20, Theorem 2], where stability is only stated as an asymptotic property, or in other words, that it holds for all large enough $n$.

The convergence of the parameters in (3.1), implies that if the limiting value $\varrho=\lim _{n \rightarrow \infty} \varrho^{n}$ is positive, then $\theta_{0}(n)$ and $C_{0}(\varepsilon)$ can be selected independent of $n$, in a manner that (3.17) holds for all sufficiently large $n$. Analogous conclusions can be drawn for Theorems 3.2 and 3.3 and Corollary 3.1 which appear later in this section.

Note also that the difference in the constant multiplying the Lyapunov function between (2.28) and (3.17) is only due to the bound in (3.10).

For the proof of the Theorem 3.1 we need the following result.

LEMma 3.1. With $\widehat{v}^{n}(x, s):=\widehat{G}^{n}(x, s)+\widehat{V}^{n}(x)$, we have the following inequality

$$
\begin{aligned}
\mathcal{A}_{z}^{n} \widehat{\mathcal{V}}^{n}(x, s) \leq \sum_{i \in \mathcal{I}}\left(\frac{\varrho^{n} \mu_{i}^{n}}{m}+\mu_{i}^{n} \hat{z}_{i}+\gamma_{i}^{n} \hat{q}_{i}(x, z)\right) \sqrt{n} \mathfrak{d} \widehat{V}^{n}\left(x ;-e_{i}\right) \\
+\varepsilon(\varepsilon+\theta) \frac{1}{\sqrt{n}} \widetilde{C}_{0}^{n} \sum_{i \in \mathcal{I}} \gamma_{i}^{n} \hat{q}_{i}(x, z) \widehat{V}^{n}(x)+\varepsilon(\varepsilon+\theta) \widetilde{C}_{1}^{n} \widehat{V}^{n}(x),
\end{aligned}
$$

with $\widetilde{C}_{0}^{n}$ and $\widetilde{C}_{1}^{n}$ as defined in (3.15).

Proof. Recall the definitions in (3.11), and note that

$$
\widehat{G}_{i}^{n}(x, s)=\left(1-\zeta_{i}^{n}\left(s_{i}\right)\right) \mathfrak{d} \widehat{V}^{n}\left(x ; e_{i}\right),
$$

with $\widehat{V}^{n}$ as defined in (3.11). It follows by direct differentiation that

$$
\frac{\mathrm{d} \zeta_{i}^{n}(\tau)}{\mathrm{d} \tau}-r_{i}^{n}(\tau) \zeta_{i}^{n}(\tau)=-\lambda_{i}^{n}, \quad \tau \geq 0
$$


Thus, using (3.13), (3.14), and (3.19), and noting that $\zeta_{i}^{n}(0)=1$, we obtain

$$
\begin{aligned}
\mathcal{A}_{z}^{n} \widehat{G}_{i}^{n}(x, s)= & -\left(\frac{\mathrm{d} \zeta_{i}^{n}\left(s_{i}\right)}{\mathrm{d} s_{i}}+r_{i}^{n}\left(s_{i}\right)\left(1-\zeta_{i}^{n}\left(s_{i}\right)\right)\right) \mathfrak{d} \widehat{V}^{n}\left(x ; e_{i}\right) \\
+ & r_{i}^{n}\left(s_{i}\right)\left(1-\zeta_{i}^{n}\left(s_{i}\right)\right) \sum_{j \neq i, i \in \mathcal{I}}\left(\mathfrak{d} \widehat{V}^{n}\left(x+e_{j} ; e_{i}\right)-\mathfrak{d} \widehat{V}^{n}\left(x ; e_{i}\right)\right) \\
& -\left(\mu_{i}^{n} z_{i}+\gamma_{i}^{n} q_{i}(x, z)\right)\left(1-\zeta_{i}^{n}\left(s_{i}\right)\right) \sum_{j \in \mathcal{I}}\left(\mathfrak{d} \widehat{V}^{n}\left(x-e_{j} ; e_{i}\right)-\mathfrak{d} \widehat{V}^{n}\left(x ; e_{i}\right)\right) \\
\leq & \left(\lambda_{i}^{n}-r_{i}^{n}\left(s_{i}\right)\right) \mathfrak{d} \widehat{V}^{n}\left(x ; e_{i}\right)+(m-1)\left(\widehat{C}_{0}^{n}\right)^{2} \widehat{C}_{1} \varepsilon(\varepsilon+\theta) \widehat{V}^{n}(x) \\
& +\frac{m}{n} \widehat{C}_{0}^{n} \widehat{C}_{1} \varepsilon(\varepsilon+\theta)\left(\mu_{i}^{n} z_{i}+\gamma_{i}^{n} q_{i}(x, z)\right) \widehat{V}^{n}(x) .
\end{aligned}
$$

Also,

$$
\mathcal{A}_{z}^{n} \widehat{V}^{n}(x)=\sum_{i \in \mathcal{I}} r_{i}^{n}\left(s_{i}\right) \mathfrak{d} \widehat{V}^{n}\left(x ; e_{i}\right)+\sum_{i \in \mathcal{I}}\left(\mu_{i}^{n} z_{i}+\gamma_{i}^{n} q_{i}(x, z)\right) \mathfrak{d} \widehat{V}^{n}\left(x ;-e_{i}\right)
$$

Applying the identities

$$
z_{i}=\sqrt{n} \hat{z}_{i}+\frac{\lambda_{i}^{n}}{\mu_{i}^{n}}+\sqrt{n} \frac{\varrho^{n}}{m}, \quad \text { and } \quad q_{i}(x, z)=\sqrt{n} \hat{q}_{i}(x, z)
$$

to $(3.21)$, we obtain

$$
\begin{aligned}
\mathcal{A}_{z}^{n} \widehat{V}^{n}(x)=\sum_{i \in \mathcal{I}} & \left(r_{i}^{n}\left(s_{i}\right) \mathfrak{d} \widehat{V}^{n}\left(x ; e_{i}\right)+\lambda_{i}^{n} \mathfrak{d} \widehat{V}^{n}\left(x ;-e_{i}\right)\right) \\
& +\sum_{i \in \mathcal{I}}\left(\frac{\varrho^{n} \mu_{i}^{n}}{m}+\mu_{i}^{n} \hat{z}_{i}+\gamma_{i}^{n} \hat{q}_{i}(x, z)\right) \sqrt{n} \mathfrak{d} \widehat{V}^{n}\left(x ;-e_{i}\right) .
\end{aligned}
$$

Combining (3.20) and (3.23), and applying once more the estimate in (3.13) and the inequality $\left|z_{i}\right| \leq n$, we deduce that

$$
\begin{aligned}
\mathcal{A}_{z}^{n} \widehat{\mathcal{V}}^{n}(x, s) \leq \sum_{i \in \mathcal{I}}\left(\frac{\varrho^{n} \mu_{i}^{n}}{m}\right. & \left.+\mu_{i}^{n} \hat{z}_{i}+\gamma_{i}^{n} \hat{q}_{i}(x, z)\right) \sqrt{n} \mathfrak{d} \widehat{V}^{n}\left(x ;-e_{i}\right) \\
& +\varepsilon(\varepsilon+\theta) \frac{m^{2}}{\sqrt{n}} \widehat{C}_{0}^{n} \widehat{C}_{1} \gamma_{i}^{n} \hat{q}_{i}(x, z) \widehat{V}^{n}(x) \\
& +\varepsilon(\varepsilon+\theta) \widehat{C}_{1}\left(m^{2} \widehat{C}_{0}^{n} \mu_{i}^{n}+m(m-1)\left(\widehat{C}_{0}^{n}\right)^{2}+\sum_{i \in \mathcal{I}} \frac{\lambda_{i}^{n}}{n}\right) \widehat{V}^{n}(x) .
\end{aligned}
$$

This completes the proof.

Proof of Theorem 3.1. The proof relies on comparing the right hand side of (3.18) to the drift inequalities in Lemma 2.1. First we fix $n \in \mathbb{N}$, and as done earlier, we suppress the $n$-dependence of $\hat{x}_{i}^{n}, \hat{z}_{i}^{n}$, and $\hat{q}_{i}^{n}$ in the calculations, in the interest of simplifying the notation. It is clear from (3.1) and (3.7) that $\hat{q}_{i} \geq 0$ if $x_{i} \geq n$, or equivalently, if

$$
\hat{x}_{i} \geq \vartheta_{n}:=\sqrt{n}\left(1-\rho_{i}^{n}\right)-\frac{\sqrt{n}}{m}\left(1-\sum_{i=1}^{m} \frac{\lambda_{i}^{n}}{n \mu_{i}^{n}}\right) \geq 0 .
$$


If $\varepsilon \leq 1$, then $\psi_{\varepsilon}(x-y)-\psi_{\varepsilon}(x) \leq-\varepsilon \frac{y}{2}$ and $\psi(-x-y)-\psi(y) \leq y$ for all $x \geq 0$ and $y \in[0,1]$ by Definition 2.1. Thus, if $\theta \in(0,1 / 2]$ and $\varepsilon \in(0,1]$, then $V(x-y) \leq V(x)$ for all $x \geq 0$ and $y \in[0,1]$. This of course implies, since $\theta_{0}(n)<1 / 2$, that $\mathfrak{d} \widehat{V}^{n}\left(x ;-e_{i}\right) \leq 0$ if $\hat{x}_{i} \geq 0$. Thus, if we write

$$
\begin{aligned}
\left(\frac{\varrho^{n} \mu_{i}^{n}}{m}+\mu_{i}^{n} \hat{z}_{i}+\gamma_{i}^{n} \hat{q}_{i}(x, z)\right) & \sqrt{n} \mathfrak{d} \widehat{V}^{n}\left(x ;-e_{i}\right) \\
= & \left(\frac{\varrho^{n} \mu_{i}^{n}}{m}+\mu_{i}^{n} \hat{z}_{i}+\gamma_{i}^{n} \hat{q}_{i}(x, z) \mathbf{1}_{\left\{\hat{x}_{i}<\vartheta_{n}\right\}}\right) \sqrt{n} \mathfrak{d} \widehat{V}^{n}\left(x ;-e_{i}\right) \\
& \quad+\gamma_{i}^{n} \hat{q}_{i}(x, z) \mathbf{1}_{\left\{\hat{x}_{i} \geq \vartheta_{n}\right\}} \sqrt{n} \mathfrak{d} \widehat{V}^{n}\left(x ;-e_{i}\right),
\end{aligned}
$$

then the second term on the right-hand side of (3.24) is negative. It is also clear that

$$
\left|\frac{\varrho^{n} \mu_{i}^{n}}{m}+\mu_{i}^{n} \hat{z}_{i}+\gamma_{i}^{n} \hat{q}_{i}(x, z) \mathbf{1}_{\left\{\hat{x}_{i}<\vartheta_{n}\right\}}\right| \leq \widehat{C}_{2}^{n} \sqrt{n}
$$

for some constant $\widehat{C}_{2}^{n}$ depending on the parameters.

Using the identity

$$
\widehat{V}^{n}\left(x \pm e_{i}\right)-\widehat{V}^{n}(x) \mp \partial_{x_{i}} \widehat{V}^{n}(x)=\int_{0}^{1}(1-t) \partial_{x_{i} x_{i}} \widehat{V}^{n}\left(x \pm t e_{i}\right) \mathrm{d} t
$$

we deduce that

$$
\left|\widehat{V}^{n}\left(x \pm e_{i}\right)-\widehat{V}^{n}(x) \mp \partial_{x_{i}} \widehat{V}^{n}(x)\right| \leq \frac{1}{n} \varepsilon(\varepsilon+\theta) \widehat{C}_{1} \widehat{V}^{n}(x),
$$

where, we use a common constant to satisfy (3.13) and (3.27). Thus, by (3.24), (3.25), and (3.27), and using also the identity

$$
\partial_{x_{i}} \widehat{V}^{n}(x)=\frac{1}{\sqrt{n}} \partial_{\hat{x}_{i}} V(\hat{x})
$$

we obtain

$$
\begin{aligned}
& \left(\frac{\varrho^{n} \mu_{i}^{n}}{m}+\mu_{i}^{n} \hat{z}_{i}+\gamma_{i}^{n} \hat{q}_{i}(x, z)\right) \sqrt{n} \mathfrak{d} \widehat{V}^{n}\left(x ;-e_{i}\right) \\
& =-\left(\frac{\varrho^{n} \mu_{i}^{n}}{m}+\mu_{i}^{n} \hat{z}_{i}+\gamma_{i}^{n} \hat{q}_{i}(x, z) \mathbf{1}_{\left\{\hat{x}_{i}<\vartheta_{n}\right\}}\right) \partial_{\hat{x}_{i}} V(\hat{x}) \\
& \quad+\gamma_{i}^{n} \hat{q}_{i}(x, z) \mathbf{1}_{\left\{\hat{x}_{i} \geq \vartheta_{n}\right\}} \sqrt{n} \mathfrak{d} \widehat{V}^{n}\left(x ;-e_{i}\right)+\varepsilon(\varepsilon+\theta) \widehat{C}_{1} \widehat{C}_{2}^{n} \widehat{V}^{n}(x) .
\end{aligned}
$$

Similarly, addressing the second term on the right-hand side of (3.18), we write

$$
\frac{1}{\sqrt{n}} \widetilde{C}_{0}^{n} \gamma_{i}^{n} \hat{q}_{i}(x, z) \leq \widehat{C}_{3}^{n}+\frac{1}{\sqrt{n}} \widetilde{C}_{0}^{n} \gamma_{i}^{n} \hat{q}_{i}(x, z) \mathbf{1}_{\left\{\hat{x}_{i} \geq \vartheta_{n}\right\}}
$$

for some constant $\widehat{C}_{3}^{n}$. Using (3.12), (3.28), and (3.29), we deduce from (3.18) that

$$
\begin{aligned}
\widehat{\mathcal{A}}_{z}^{n} \mathcal{V}^{n}(\hat{x}, s) \leq- & \sum_{i \in \mathcal{I}}\left(\frac{\varrho^{n} \mu_{i}^{n}}{m}+\mu_{i}^{n} \hat{z}_{i}+\gamma_{i}^{n} \hat{q}_{i}(\hat{x}, \hat{z}) \mathbf{1}_{\left\{\hat{x}_{i}<\vartheta_{n}\right\}}\right) \partial_{\hat{x}_{i}} V(\hat{x}) \\
+ & \varepsilon(\varepsilon+\theta)\left(\widetilde{C}_{1}^{n}+m \widehat{C}_{1} \widehat{C}_{2}^{n}+m \widehat{C}_{3}^{n}\right) V(\hat{x}) \\
& +\sum_{i \in \mathcal{I}}\left(\sqrt{n} \mathfrak{d} \widehat{V}^{n}\left(x ;-e_{i}\right)+\varepsilon(\varepsilon+\theta) \frac{1}{\sqrt{n}} \widetilde{C}_{0}^{n} \widehat{V}^{n}(x)\right) \gamma_{i}^{n} \hat{q}_{i}(\hat{x}, \hat{z}) \mathbf{1}_{\left\{\hat{x}_{i} \geq \vartheta_{n}\right\}},
\end{aligned}
$$

where we express $\hat{q}$ as a function of $\hat{x}$ and $\hat{z}$, slightly abusing the notation. 
We now turn to the drift inequalities in Lemma 2.1. It follows by (2.17) that there exists a constant and $C_{0}^{n}(\varepsilon)$, such that

$$
\begin{aligned}
\sum_{i \in \mathcal{I}}\left(-\frac{\varrho^{n} \mu_{i}^{n}}{m}-\mu_{i}^{n}\left(\hat{x}_{i}-\langle e, \hat{x}\rangle^{+} u_{i}\right)-\gamma_{i}^{n}\langle e, \hat{x}\rangle^{+} u_{i} \mathbf{1}_{\left\{\hat{x}_{i}<\vartheta_{n}\right\}}\right) \partial_{\hat{x}_{i}} V(\hat{x}) \\
+\varepsilon(\varepsilon+\theta)\left(\widetilde{C}_{1}^{n}+m \widehat{C}_{1} \widehat{C}_{2}^{n}+m \widehat{C}_{3}^{n}\right) V(\hat{x}) \leq C_{0}^{n}(\varepsilon)-\varepsilon \frac{\varrho^{n}}{2 m} V(\hat{x})
\end{aligned}
$$

for all $(\hat{x}, u) \in \mathbb{R}^{m} \times \Delta$, and for all $\varepsilon \in\left(0, \theta_{0}(n)\right)$.

Consider the first sum in (3.31). If $\langle e, x\rangle \leq n$, then $\hat{z}=\hat{x}$ by work-conservation. Note also that by the scaling in (3.3) combined with (3.2), we have

$$
\langle e, \hat{x}\rangle=\frac{1}{\sqrt{n}}(\langle e, x\rangle-n) .
$$

Thus $\langle e, \hat{x}\rangle>0$ if and only if $\langle e, x\rangle>n$. Similarly $\langle e, z\rangle=n$ if and only if $\langle e, \hat{z}\rangle=0$. On the other hand, if $\langle e, x-z\rangle>0$, then we can write $z=x-\langle e, x-z\rangle u$, for some $u \in \Delta$. Thus, $\hat{z}=\hat{x}-\langle e, \hat{x}-$ $\hat{z}\rangle u=\hat{x}-\langle e, \hat{x}\rangle u$, since $\langle e, \hat{z}\rangle=0$. We have thus established that for all $x \in \mathbb{R}_{+}^{m}$, we have

$$
\hat{z}=\hat{x}-\langle e, \hat{x}\rangle^{+} u, \quad \text { and } \hat{q}(\hat{x}, \hat{z})=\langle e, \hat{x}\rangle^{+} u
$$

for some $u \in \Delta$. It then follows from (3.32), that the sum of the first two terms on the right-hand of (3.30) has the bound on the right-hand side in (3.31).

Next, consider the last term in (3.30). By (3.26) and (3.27) we have

$$
\mathfrak{d} \widehat{V}^{n}\left(x ;-e_{i}\right) \leq-\partial_{x_{i}} \widehat{V}^{n}(x)+\frac{1}{n} \varepsilon(\varepsilon+\theta) \widehat{C}_{1} \widehat{V}^{n}(x),
$$

and $\partial_{x_{i}} \widehat{V}^{n}(x)=\frac{\varepsilon}{\sqrt{n} \mu_{i}^{n}} \widehat{V}^{n}(x)$ when $\hat{x}_{i} \geq \vartheta_{n}$. Thus

$$
\left(\sqrt{n} \mathfrak{d} \widehat{V}^{n}\left(x ;-e_{i}\right)+\varepsilon(\varepsilon+\theta) \frac{1}{\sqrt{n}} \widetilde{C}_{0}^{n} \widehat{V}^{n}(x)\right) \mathbf{1}_{\left\{\hat{x}_{i} \geq \vartheta_{n}\right\}} \leq-\varepsilon\left(\frac{1}{\mu_{i}^{n}}-(\varepsilon+\theta) \frac{1}{\sqrt{n}}\left(\widetilde{C}_{0}^{n}+\widehat{C}_{1}\right)\right) \widehat{V}^{n}(x),
$$

which is negative for all $\varepsilon<\theta=\theta_{0}(n)$, by the definition of $\theta_{0}$ in (3.16). Thus, in view of (3.10), we have established the Foster-Lyapunov equation (3.17) as claimed.

The remaining conclusions of the theorem are straightforward, in view of the fact that $\left\{S^{n}(t)\right\}_{t \geq 0}$ is positive Harris recurrent, as shown in [28]. Note that since $\varepsilon<\tilde{\varepsilon}_{0}(\theta)$, then (3.10) implies that $\mathcal{V}^{n}$ is bounded from below in $\mathbb{R}^{m} \times \mathbb{R}_{+}^{m}$.

In the theorem that follows we assume strictly positive abandonment rates for all classes, and we use the Lyapunov function

$$
\mathcal{V}^{n}\left(\hat{x}^{n}, s\right):=\sum_{i \in \mathcal{I}}\left(1-\zeta_{i}^{n}\left(s_{i}\right)\right)\left(\varphi^{n}\left(\hat{x}_{i}^{n}+n^{-1 / 2}\right)-\varphi^{n}\left(\hat{x}_{i}^{n}\right)\right)+\sum_{i \in \mathcal{I}} \frac{\varphi^{n}\left(\hat{x}_{i}^{n}\right)}{\mu_{i}^{n}},
$$

with

$$
\varphi^{n}(y):=\tilde{\varepsilon}_{0}\left(\theta^{n}\right) \theta^{n} \psi(-y)+\tilde{\varepsilon}_{0}\left(\theta^{n}\right) \psi(y), \quad y \in \mathbb{R},
$$

$\tilde{\varepsilon}_{0}$ as in $(3.9)$, and

$$
\theta^{n}=1 \wedge \frac{\left(1-\beta_{\min }^{n}\right) \vee \frac{1}{2}}{\beta_{\max }^{n}}, \quad \beta_{i}^{n}:=\frac{\gamma_{i}^{n}}{\mu_{i}^{n}}, \quad i \in \mathcal{I} .
$$


A. Arapostathis, H. Hmedi and G. Pang: Uniform exponential ergodicity of multiclass many-server queues

Theorem 3.2. Grant Assumption 3.1, and, in addition, assume that hazard rate functions $\left\{h_{i}\right\}_{i \in \mathcal{I}}$ are locally bounded. Suppose $\gamma_{i}^{n}>0$ for all $i \in \mathcal{I}$. Then there exist positive constants $c_{0}(n)$ and $c_{1}(n)$, depending only on $n \in \mathbb{N}$, such that the function $\mathcal{V}^{n}$ in (3.33) satisfies

$$
\widehat{\mathcal{A}}_{z}^{n} \mathcal{V}^{n}(\hat{x}, s) \leq c_{0}(n)-c_{1}(n) \mathcal{V}^{n}(\hat{x}, s) \quad \forall(\hat{x}, s) \in X^{n} \times \mathbb{R}_{+}^{m}, \forall z \in \mathcal{Z}^{n}(\hat{x}) .
$$

In particular, under any work-conserving stationary Markov policy, the process $\left(\hat{X}^{n}, S^{n}\right)$ is positive Harris recurrent, and $\|\hat{x}\|_{1}$ is integrable under its invariant probability distribution.

Proof. The proof mimics that of Theorem 3.1 also using Remark 2.6. The important difference here is that, if we let $\hat{\varphi}^{n}\left(x_{i}\right):=\varphi^{n}\left(\hat{x}_{i}^{n}\left(x_{i}\right)\right)$, and

$$
\hat{\phi}_{i}^{n}(x, s):=\left(1-\zeta_{i}^{n}\left(s_{i}\right)\right) \mathfrak{d} \hat{\varphi}^{n}\left(x_{i} ; 1\right),
$$

then, following the steps in (3.20), we obtain

$$
\begin{aligned}
\mathcal{A}_{z}^{n} \hat{\phi}_{i}^{n}(x, s)=\left(\lambda_{i}^{n}-r_{i}^{n}\left(s_{i}\right)\right) \mathfrak{d} \hat{\varphi}^{n}\left(x_{i} ; 1\right) & \\
& -\left(\mu_{i}^{n} z_{i}+\gamma_{i}^{n} q_{i}(x, z)\right)\left(1-\zeta_{i}^{n}\left(s_{i}\right)\right)\left(\mathfrak{d} \phi^{n}\left(x_{i}-1 ; 1\right)-\mathfrak{d} \phi^{n}\left(x_{i} ; 1\right)\right) .
\end{aligned}
$$

As a result, the terms corresponding to the second line of (3.20), for which the assumption that the hazard rate functions are bounded was invoked, are not present in (3.35). The rest of the proof is the same.

Without assuming that the abandonment rates are positive, but with $\varrho^{n}>0$, we obtain uniform stability, that is, tightness of the invariant distributions. To establish this, we scale the Lyapunov function in (3.33), with a parameter $\varepsilon>0$. More precisely, we define

$$
\mathcal{V}_{\varepsilon}^{n}(\hat{x}, s):=\sum_{i \in \mathcal{I}}\left(1-\zeta_{i}^{n}\left(s_{i}\right)\right) \mathfrak{d} \varphi_{\varepsilon}^{n}\left(\hat{x}_{i} ; n^{-1 / 2}\right)+\sum_{i \in \mathcal{I}} \frac{\varphi_{\varepsilon}^{n}\left(\hat{x}_{i}\right)}{\mu_{i}^{n}}
$$

with

$$
\varphi_{\varepsilon}^{n}(y):=\theta^{n} \varepsilon \psi(-y)+\psi_{\varepsilon}(y), \quad y \in \mathbb{R} .
$$

The parameter $\theta^{n}$ depends on certain bounds which we review next. First, as we have seen in (3.13), there is a constant $\check{C}_{1}$ such that

$$
\left|\mathfrak{d} \varphi_{\varepsilon}^{n}\left(x \pm n^{-1 / 2} e_{j} ; \pm n^{-1 / 2} e_{i}\right)-\mathfrak{d} \varphi_{\varepsilon}^{n}\left(x ; \pm n^{-1 / 2} e_{i}\right)\right| \leq \frac{1}{n} \check{C}_{1} \varepsilon(\varepsilon+\theta) \quad \forall i, j \in \mathcal{I} .
$$

Let also $\check{C}_{0}^{n}$ be a bound for $\left\|\max _{i} \zeta_{i}^{n}\right\|_{\infty}$. With $\widehat{C}_{2}^{n}$ the constant in (3.25), we define

$$
\bar{C}_{0}^{n}:=m^{2} \check{C}_{0}^{n} \check{C}_{1}, \quad \text { and } \quad \bar{C}_{1}^{n}:=\check{C}_{1}\left(m^{2} \check{C}_{0}^{n} \mu_{i}^{n}+\sum_{i \in \mathcal{I}} \frac{\lambda_{i}^{n}}{n}\right) .
$$

Let $\theta^{n}$ be equal to the right-hand side of (3.16) after we replace $\widehat{C}_{1}, \widetilde{C}_{1}^{n}$, and $\widetilde{C}_{2}^{n}$ with $\check{C}_{1}, \bar{C}_{1}^{n}$, and $\bar{C}_{2}^{n}$, respectively.

THEOREM 3.3. Grant Assumption 3.1, and, in addition, assume that hazard rate functions $\left\{h_{i}\right\}_{i \in \mathcal{I}}$ are locally bounded. Suppose that $\varrho^{n}>0$. Then there exist a cube $K$ and a constant $C$ depending on $\varepsilon, \varrho^{n}$, and $\theta^{n}$, defined above, such that the function $\mathcal{V}_{\varepsilon}^{n}$ in (3.36) satisfies

$$
\widehat{\mathcal{A}}_{z}^{n} \mathcal{V}_{\varepsilon}^{n}(\hat{x}, s) \leq \varepsilon C \mathbf{1}_{K}(\hat{x})-\varepsilon \frac{\varrho^{n}}{3 m} \quad \forall(\hat{x}, s) \in X^{n} \times \mathbb{R}_{+}^{m}, \forall z \in \mathcal{Z}^{n}(\hat{x}),
$$

and for all $\varepsilon \leq \theta^{n}$. In particular, under any work-conserving stationary Markov policy, the process $\left(\hat{X}^{n}, S^{n}\right)$ is positive Harris recurrent.

Proof. We follow the proofs of Lemma 3.1 and Theorem 3.1 to obtain the analogous inequality to (3.30). The result then follows by applying the drift inequality in (2.17), and using the definition of $\theta^{n}$. 
3.3. Results with Poisson arrivals. In this subsection, we specialize the results to a sequence of queueing models with Poisson arrivals with rates $\lambda_{i}^{n}, i \in \mathcal{I}$. Here, under a stationary Markov policy, the process $\left\{X^{n}(t)\right\}_{t \geq 0}$ is Markov with extended generator

$$
\mathcal{A}_{z}^{n} f(x):=\sum_{i \in \mathcal{I}} \lambda_{i}^{n}\left(f\left(x+e_{i}\right)-f(x)\right)+\sum_{i \in \mathcal{I}}\left(\mu_{i}^{n} z_{i}+\gamma_{i}^{n} q_{i}(x, z)\right)\left(f\left(x-e_{i}\right)-f(x)\right) .
$$

Define $\widehat{\mathcal{A}}_{z}^{n}$ analogously to (3.12). Mimicking the proof of Theorem 3.1, we deduce the following, which we state without proof.

Corollary 3.1. Assume that the arrival processes are Poisson. Suppose $\varrho^{n}>0$. Then for some $\theta=\theta(n)>0$, there exist positive constants $\hat{\varepsilon}_{0}(n)$ and $C_{0}^{n}(\varepsilon)$, such that the function $V$ in (2.16) satisfies

$$
\widehat{\mathcal{A}}_{z}^{n} V(\hat{x}) \leq C_{0}^{n}(\varepsilon)-\varepsilon \frac{\varrho^{n}}{2 m} V(\hat{x}) \quad \forall \hat{x} \in \mathcal{X}^{n}, \forall z \in \mathcal{Z}^{n}(\hat{x})
$$

and for all $\varepsilon \in\left(0, \hat{\varepsilon}_{0}(n)\right)$. In particular, under any work-conserving stationary Markov policy, the process $\left\{\hat{X}^{n}(t)\right\}_{t \geq 0}$ is exponentially ergodic, and $V(\hat{x})$ is integrable under its invariant probability measure.

REMARK 3.3. Let $\mathfrak{Z}_{\mathrm{sm}}^{n}$ denote the class of work-conserving stationary Markov policies for the process $\hat{X}^{n}(t)$. Suppose $\varrho>0$, and let $P_{t}^{n, z}$ and $\pi_{z}^{n}$ denote the transition probability and the stationary distribution, respectively, of $\hat{X}^{n}(t)$ under a policy $z \in \mathfrak{Z}_{\mathrm{sm}}^{n}$. Then, Corollary 3.1 implies that there exist positive constants $\gamma$ and $C_{\gamma}$ not depending on $n$ or $z$, such that

$$
\left\|P_{t}^{n, z}(\hat{x}, \cdot)-\pi_{z}^{n}(\cdot)\right\|_{V} \leq C_{\gamma} V(\hat{x}) \mathrm{e}^{-\gamma t}, \quad \forall \hat{x} \in X^{n}, \forall t \geq 0 .
$$

Also

$$
\sup _{n \in \mathbb{N}} \sup _{z \in \mathfrak{J}_{\mathrm{sm}}^{n}} \int_{X^{n}} V(\hat{x}) \pi_{z}^{n}(\mathrm{~d} \hat{x})<\infty .
$$

Note that, if $\nu^{n}$ denotes the distribution of $\hat{X}^{n}(0)$, then (3.38) implies that

$$
\left\|P_{t}^{n, z}\left(\nu^{n}, \cdot\right)-\pi_{z}^{n}(\cdot)\right\|_{V} \leq C_{\gamma} \nu^{n}(V) \mathrm{e}^{-\gamma t} \quad \forall t \geq 0,
$$

where $P_{t}^{n, z}\left(\nu^{n}, \cdot\right):=\int_{X^{n}} \nu^{n}(\mathrm{~d} \hat{x}) P_{t}^{n, z}(\hat{x}, \cdot)$ and $\nu^{n}(V):=\int_{X^{n}} V(\hat{x}) \nu^{n}(\mathrm{~d} \hat{x})$. In particular, if $\hat{X}^{n}(0)$ is such that $\sup _{n \in \mathbb{N}} \nu^{n}(V)<\infty$, then the convergence in (3.39) is uniform over $z \in \mathfrak{Z}_{\mathrm{sm}}^{n}$ and $n \in \mathbb{N}$.

We also wish to remark that, provided that the jobs do not abandon the queues, that is, $\Gamma=0$, the hypothesis $\varrho^{n}>0$ is sharp. In fact, there is a dichotomy. As shown in Corollary 3.1, if $\varrho^{n}>0$, then $\left\{X^{n}(t)\right\}_{t \geq 0}$ is uniformly exponentially ergodic. Following for example the proof in [9, Theorem 3.3] one can show that if $\varrho^{n}<0$ and jobs do not abandon the queues, then $\left\{X^{n}(t)\right\}_{t \geq 0}$ is transient under any Markov scheduling policy.

As explained in [20, p. 33], under positive abandonment in all classes, the invariant distribution of $\hat{X}^{n}$ cannot integrate a function of the form $\mathrm{e}^{\varepsilon|\hat{x}|^{2}}$ for $\varepsilon>0$, even though the invariant probability distribution of the limit diffusion has this property as seen in Theorem 2.2. Note that the technique in the proof of Theorem 3.1 stumbles in (3.27), since this bound is no longer valid for the function $\widetilde{V}$ of Theorem 2.2 .

Nevertheless, we have the following improvement of Corollary 3.1, under positive abandonment in all classes.

Theorem 3.4. Assume that the arrival processes are Poisson. Suppose $\liminf _{n \rightarrow \infty} \gamma_{i}^{n}>0$ for all $i \in \mathcal{I}$. Then there exist positive constants $\breve{\kappa}_{0}(\eta)$ and $\breve{\kappa}_{1}(\eta)$, such that the function

$$
\breve{V}^{n}(\hat{x}):=\exp \left(\Phi_{\eta, \theta^{n}}^{*}(\hat{x})\right)=\exp \left(\eta \theta^{n} \Psi(-\hat{x})+\eta \Psi(\hat{x})\right)
$$


with $\theta^{n}$ given by (3.34), satisfies

$$
\widehat{\mathcal{A}}_{z}^{n} \breve{V}^{n}(\hat{x}) \leq \breve{\kappa}_{0}(\eta)-\breve{\kappa}_{1}(\eta)\|\hat{x}\|_{1} \breve{V}^{n}(\hat{x}) \quad \forall(\hat{x}, z) \in X^{n} \times \mathcal{Z}^{n}(\hat{x}),
$$

and for all sufficiently large $n$. In particular, the function $\exp \left(\eta\left\|\hat{x}^{n}\right\|_{1}\right)$ is integrable under the stationary distribution of $\left\{\hat{X}^{n}(t)\right\}_{t \geq 0}$ for all $\eta>0$, under any work-conserving stationary Markov scheduling policy.

Proof. Let $\widehat{\mathcal{V}}^{n}(x):=\breve{V}\left(\hat{x}^{n}(x)\right)$. Applying the operator in (3.37) to $\widehat{\mathcal{V}}^{n}$ and using the analogous bound to (3.27),

$$
\begin{aligned}
\mathcal{A}_{z}^{n} \widehat{\mathcal{V}}^{n}(x) \leq \sum_{i \in \mathcal{I}}\left[\lambda _ { i } ^ { n } \left(\partial_{x_{i}} \widehat{\mathcal{V}}^{n}(x)\right.\right. & \left.+\frac{1}{n} \eta(1+\theta) \widehat{C} \widehat{\mathcal{V}}^{n}(x)\right) \\
& \left.+\left(\mu_{i}^{n} z_{i}+\gamma_{i}^{n} q_{i}(x, z)\right)\left(-\partial_{x_{i}} \widehat{\mathcal{V}}^{n}(x)+\frac{1}{n} \eta(1+\theta) \widehat{C} \widehat{\mathcal{V}}^{n}(x)\right)\right]
\end{aligned}
$$

for some constant $\widehat{C}$. Using $(3.22)$ we write this as

$$
\begin{aligned}
\widehat{\mathcal{A}}_{z}^{n} \breve{V}(\hat{x}) \leq \sum_{i \in \mathcal{I}}\left(-\frac{\varrho^{n} \mu_{i}^{n}}{d}-\mu_{i}^{n} \hat{z}_{i}-\gamma_{i}^{n} \hat{q}_{i}(\hat{x}, \hat{z})\right) \partial_{\hat{x}_{i}} \breve{V}(\hat{x}) \\
\quad+\eta(1+\theta) \widehat{C} \sum_{i \in \mathcal{I}}\left(\frac{\lambda_{i}^{n}}{n}+\frac{\mu_{i}^{n}}{n} z_{i}+\frac{1}{\sqrt{n}} \gamma_{i}^{n}\left(\hat{x}_{i}-\hat{z}_{i}\right)\right) \breve{V}(\hat{x}) .
\end{aligned}
$$

Thus, using the drift inequality in Remark 2.6 to bound the first term on the right-hand side of (3.40), and noting that the coefficient of $\breve{V}$ on the second term on the right-hand side is of order $\frac{1}{\sqrt{n}}\|x\|_{1}$, we establish the result.

We conclude with the analogous result to Corollary 2.1. We need the following notation.

$$
\hat{\mathcal{I}}_{1}:=\left\{i \in \mathcal{I}: \limsup _{n \rightarrow \infty} \frac{\gamma_{i}^{n}}{\mu_{i}^{n}}<1\right\} .
$$

Theorem 3.5. Assume that the arrival processes are Poisson. Suppose $\liminf _{n \rightarrow \infty} \varrho^{n}>0$. Then the function $\exp \left(\eta \sum_{i \in \hat{\mathcal{I}}_{1}} \hat{x}_{i}^{n}\right)$ is integrable under the invariant probability distribution of $\left\{\hat{X}^{n}(t)\right\}_{t \geq 0}$ for all $\eta>0$, and for all sufficiently large $n$.

The proof closely mimics that of Theorem 3.1, and is therefore omitted.

Acknowledgment. This work was supported in part by an Army Research Office grant W911NF-17-1-0019, in part by NSF grants DMS-1715210, CMMI-1635410, and DMS/CMMI1715875, and in part by the Office of Naval Research through grant N00014-16-1-2956.

\section{References}

[1] Aghajani R, Ramanan K (2016) The limit of stationary distributions of many-server queues in the Halfin-Whitt regime. arXiv eprints https://arxiv.org/abs/1610.01118.

[2] Aghajani R, Ramanan K (2019) Ergodicity of an SPDE associated with a many-server queue. Ann. Appl. Probab. 29(2):994-1045, http://dx.doi.org/10.1214/18-AAP1419.

[3] Arapostathis A, Biswas A, Pang G (2015) Ergodic control of multi-class $M / M / N+M$ queues in the Halfin-Whitt regime. Ann. Appl. Probab. 25(6):3511-3570, http://dx.doi.org/10.1214/14-AAP1081.

[4] Arapostathis A, Borkar VS, Ghosh MK (2011) Ergodic control of diffusion processes, volume 143 of Encyclopedia of Mathematics and its Applications (Cambridge: Cambridge University Press), http://dx.doi.org/10.1017/CB09781139003605. 
[5] Arapostathis A, Borkar VS, Kumar KS (2014) Convergence of the relative value iteration for the ergodic control problem of nondegenerate diffusions under near-monotone costs. SIAM J. Control Optim. 52(1):1-31, http://dx.doi.org/10.1137/130912918.

[6] Arapostathis A, Pang G (2016) Ergodic diffusion control of multiclass multi-pool networks in the HalfinWhitt regime. Ann. Appl. Probab. 26(5):3110-3153, http://dx.doi.org/10.1214/16-AAP1171.

[7] Arapostathis A, Pang G (2018) Infinite-horizon average optimality of the N-network in the Halfin-Whitt regime. Math. Oper. Res. 43(3):838-866, http://dx.doi.org/10.1287/moor.2017.0886.

[8] Arapostathis A, Pang G (2019) Infinite horizon asymptotic average optimality for large-scale parallel server networks. Stochastic Process. Appl. 129(1):283-322, http://dx.doi.org/10.1016/j.spa.2018.03.005.

[9] Arapostathis A, Pang G, Sandrić N (2019) Ergodicity of a Lévy-driven SDE arising from multiclass many-server queues. Ann. Appl. Probab. 29(2):1070-1126, http://dx.doi.org/10.1214/18-AAP1430.

[10] Atar R, Mandelbaum A, Reiman MI (2004) Scheduling a multi class queue with many exponential servers: asymptotic optimality in heavy traffic. Ann. Appl. Probab. 14(3):1084-1134, http://dx.doi.org/10.1214/105051604000000233.

[11] Braverman A, Dai JG (2017) Stein's method for steady-state diffusion approximations of $M / P h / n+M$ systems. Ann. Appl. Probab. 27(1):550-581, http://dx.doi.org/10.1214/16-AAP1211.

[12] Braverman A, Dai JG, Feng J (2016) Stein's method for steady-state diffusion approximations: an introduction through the Erlang-A and Erlang-C models. Stoch. Syst. 6(2):301-366, http://dx.doi.org/10.1214/15-SSY212.

[13] Braverman A, Dai JG, Miyazawa M (2017) Heavy traffic approximation for the stationary distribution of a generalized Jackson network: the BAR approach. Stoch. Syst. 7(1):143-196, http://dx.doi.org/10.1287/15-SSY199.

[14] Budhiraja A, Lee C (2009) Stationary distribution convergence for generalized Jackson networks in heavy traffic. Math. Oper. Res. 34(1):45-56, http://dx.doi.org/10.1287/moor.1080.0353.

[15] Dai JG, Dieker AB, Gao X (2014) Validity of heavy-traffic steady-state approximations in many-server queues with abandonment. Queueing Syst. 78(1):1-29, http://dx.doi.org/10.1007/s11134-014-9394-x.

[16] Dai JG, He S (2013) Many-server queues with customer abandonment: numerical analysis of their diffusion model. Stoch. Syst. 3(1):96-146, http://dx.doi.org/10.1214/11-SSY029.

[17] Dieker AB, Gao X (2013) Positive recurrence of piecewise Ornstein-Uhlenbeck processes and common quadratic Lyapunov functions. Ann. Appl. Probab. 23(4):1291-1317, http://dx.doi.org/10.1214/12-aap870.

[18] Gamarnik D, Goldberg DA (2013) Steady-state GI/GI/n queue in the Halfin-Whitt regime. Ann. Appl. Probab. 23(6):2382-2419, http://dx.doi.org/10.1214/12-AAP905.

[19] Gamarnik D, Momčilović P (2008) Steady-state analysis of a multiserver queue in the Halfin-Whitt regime. Adv. in Appl. Probab. 40(2):548-577, http://dx.doi.org/10.1239/aap/1214950216.

[20] Gamarnik D, Stolyar AL (2012) Multiclass multiserver queueing system in the Halfin-Whitt heavy traffic regime: asymptotics of the stationary distribution. Queueing Syst. 71(1-2):25-51, http://dx.doi.org/10.1007/s11134-012-9294-x.

[21] Gamarnik D, Zeevi A (2006) Validity of heavy traffic steady-state approximation in generalized Jackson networks. Ann. Appl. Probab. 16(1):56-90, http://dx.doi.org/10.1214/105051605000000638.

[22] Gruber M (1984) Harnack inequalities for solutions of general second order parabolic equations and estimates of their Hölder constants. Math. Z. 185(1):23-43, http://dx.doi .org/10.1007/BF01214972.

[23] Gurvich I (2014) Validity of heavy-traffic steady-state approximations in multiclass queueing networks: the case of queue-ratio disciplines. Math. Oper. Res. 39(1):121-162, http://dx.doi.org/10.1287/moor.2013.0593.

[24] Gyöngy I, Krylov N (1996) Existence of strong solutions for Itô's stochastic equations via approximations. Probab. Theory Related Fields 105(2):143-158, http://dx.doi.org/10.1007/BF01203833. 
[25] Halfin S, Whitt W (1981) Heavy-traffic limits for queues with many exponential servers. Oper. Res. 29(3):567-588, http://dx.doi.org/10.1287/opre.29.3.567.

[26] Harrison JM, Zeevi A (2004) Dynamic scheduling of a multiclass queue in the Halfin-Whitt heavy traffic regime. Oper. Res. 52(2):243-257, http://dx.doi.org/10.1287/opre.1030.0084.

[27] Katsuda $\mathrm{T}$ (2010) State-space collapse in stationarity and its application to a multiclass single-server queue in heavy traffic. Queueing Syst. 65(3):237-273, http://dx.doi.org/10.1007/s11134-010-9178-x.

[28] Konstantopoulos T, Last G (1999) On the use of Lyapunov function methods in renewal theory. Stochastic Process. Appl. 79(1):165-178, http://dx.doi.org/10.1016/S0304-4149(98) 00068-4.

[29] Meyn SP, Tweedie RL (1993) Stability of Markovian processes. III. FosterLyapunov criteria for continuous-time processes. Adv. in Appl. Probab. 25(3):518-548, http://dx.doi.org/10.2307/1427522.

[30] Meyn SP, Tweedie RL (1994) Computable bounds for geometric convergence rates of Markov chains. Ann. Appl. Probab. 4(4):981-1011.

[31] Pang G, Talreja R, Whitt W (2007) Martingale proofs of many-server heavy-traffic limits for Markovian queues. Probab. Surv. 4:193-267, http://dx.doi.org/10.1214/06-PS091.

[32] Stolyar AL (2015) Diffusion-scale tightness of invariant distributions of a large-scale flexible service system. Adv. in Appl. Probab. 47(1):251-269, http://dx.doi.org/10.1239/aap/1427814590.

[33] Stolyar AL (2015) Tightness of stationary distributions of a flexible-server system in the Halfin-Whitt asymptotic regime. Stoch. Syst. 5(2):239-267, http://dx.doi.org/10.1214/14-SSY139.

[34] Stolyar AL, Yudovina E (2012) Tightness of invariant distributions of a large-scale flexible service system under a priority discipline. Stoch. Syst. 2(2):381-408, http://dx.doi.org/10.1214/12-SSY063.

[35] Stolyar AL, Yudovina E (2013) Systems with large flexible server pools: instability of "natural" load balancing. Ann. Appl. Probab. 23(5):2099-2138, http://dx.doi.org/10.1214/12-AAP895.

[36] Whitt W (1992) Understanding the efficiency of multi-server service systems. Management Science 38(5):708-723, http://dx.doi.org/10.1287/mnsc.38.5.708.

[37] Ye HQ, Yao DD (2016) Diffusion limit of fair resource control - stationarity and interchange of limits. Math. Oper. Res. 41(4):1161-1207, http://dx.doi.org/10.1287/moor.2015.0773.

[38] Ye HQ, Yao DD (2018) Justifying diffusion approximations for multiclass queueing networks under a moment condition. Ann. Appl. Probab. 28(6):3652-3697, http://dx.doi.org/10.1214/18-AAP1401. 